\title{
MORE ON THE PRESSING DOWN GAME.
}

\author{
JAKOB KELLNER* AND SAHARON SHELAH ${ }^{\dagger}$
}

\begin{abstract}
We investigate the pressing down game and its relation to the Banach Mazur game. In particular we show: Consistently, there is a nowhere precipitous normal ideal $I$ on $\aleph_{2}$ such that player nonempty wins the pressing down game of length $\aleph_{1}$ on $I$ even if player empty starts.
\end{abstract}

We investigate the pressing down game and its relation to the Banach Mazur game. Definitions (and some well known or obvious properties) are given in Section 1. The results are summarized in Section 2. This paper continues (and simplifies, see 2.2) the investigation of Pauna and the authors in [15].

We thank the referee for kindly pointing out an error and an embarrasingly large number of typos.

After the submission of this paper it came to our attention that Gitik 6 already proved Fact 6.1 of this paper, moreover he just requires a measurable cardinal (we use a supercompact). Nevertheless we include our proof in this paper, maybe the construction could be of intrerest in other situations.

\section{Definitions}

We use the following notation:

- For forcing conditions $q \leq p$, the smaller condition $q$ is the stronger one. We stick to Goldstern's alphabetic convention [8, 1.2]: Whenever two conditions are comparable the notation is chosen so that the variable used for the stronger condition comes "lexicographically" later.

- $E_{\lambda}^{\kappa}=\{\alpha \in \kappa: \operatorname{cf}(\alpha)=\lambda\}$.

- $\mathrm{NS}_{\kappa}$ is the nonstationary ideal on $\kappa$.

- The dual of an ideal $I$ is the filter $\{A \subseteq \kappa: \kappa \backslash A \in I\}$ and vice versa.

- For an ideal $I$ on $\kappa$ and a positive set $A$ (i.e., $A \notin I$ ), we set $I \uparrow A$ to be the ideal generated by $I \cup\{\kappa \backslash A\}$.

We always assume that $\kappa$ is a regular uncountable cardinal and that $I$ is a $<\kappa$ complete ideal on $\kappa$. Unless noted otherwise, we will also assume that $I$ is normal.

We now recall the definitions of several games of length $\omega$, played by the players empty and nonempty. We abbreviate "having a winning strategy for $G$ " with "winning $G$ " (as opposed to: "winning a specific run of $G$ ").

First we define four variants of the pressing down game (this game has been used, e.g., in [17]).

Date: 2006-09-14.

2000 Mathematics Subject Classification. 03E35;03E55.

* supported by European Union FP7 grant PERG02-GA-2207-224747 and the Austrian FWF project P21651-N13.

$\dagger$ supported by the United States-Israel Binational Science Foundation (Grant no. 2002323), publication 939 . 
Definition 1.1. - $\mathrm{PD}(I)$ is played as follows: Set $S_{-1}=\kappa$. At stage $n$, empty chooses a regressive function $f_{n}: \kappa \rightarrow \kappa$, and nonempty chooses $S_{n}$, an $f_{n}$-homogeneous $I$-positive subset of $S_{n-1}$. Empty wins the run of the game if $\bigcap_{n \in \omega} S_{n} \in I$.

- $\mathrm{PD}^{\emptyset}(I)$ is played like $\mathrm{PD}(I)$, but empty wins the run if $\bigcap_{n \in \omega} S_{n}=\emptyset$.

- $\mathrm{PD}_{\mathrm{e}}(I)$ is played like $\mathrm{PD}(I)$, but empty can first choose $S_{-1}$ to be an arbitrary $I$-positive set.

- $\mathrm{PD}_{\mathrm{e}}^{\emptyset}$ is defined analogously.

So we have four variants of the pressing down game, depending on two parameters: whether the winning condition for player nonempty is " $\neq \emptyset "$ or " $\notin I "$ ", and whether empty has the first move or not.

We now analogously define four variants of the Banach Mazur game:

Definition 1.2. $\quad \operatorname{BM}(I)$ is played as follows: Set $S_{-1}=\kappa$. At stage $n$, empty chooses an $I$-positive subset $X$ of $S_{n-1}$, and nonempty chooses an $I$-positive subset $S_{n}$ of $X$. Empty wins the run if $\bigcap_{n \in \omega} S_{n} \in I$.

- The ideal game $\operatorname{Id}(I)$ is played just like $\operatorname{BM}(I)$, but empty wins the run if $\bigcap_{n \in \omega} S_{n}=\emptyset$.

- $\mathrm{BM}_{\text {ne }}(I)$ is played just like $\mathrm{BM}(I)$, but nonempty has the first move.

- $\operatorname{Id}_{\text {ne }}(I)$ is defined analogously.

More generally, we can define the Banach Mazur game $\operatorname{BM}(B)$ on a Boolean algebra $B$ : The players choose decreasing (nonzero) elements $a_{n} \in B$, nonempty wins if there is some (nonzero) $b \in B$ smaller than all $a_{n}$. Then $\operatorname{BM}(I)$ is equivalent to the corresponding game $\mathrm{BM}\left(B_{I}\right)$ on the Boolean algebra $B_{I}=\mathfrak{P}(\kappa) / I$ (since $I$ is $\sigma$-complete), the same holds for $\mathrm{BM}_{\text {ne }}(I)$ and $\mathrm{BM}_{\text {ne }}\left(B_{I}\right)$; we could equivalently use the completion $\operatorname{ro}\left(B_{I}\right)$ instead of $B_{I}$. Also the $\notin I$ versions of the pressing down game can be played modulo null sets, i.e., on the Boolean algebra $B_{I}$, in the obvious way. For the $\neq \emptyset$ versions of the games, the version played on $B_{I}$ does not make sense.

In the $\notin I$ version, the pressing down and Banach Mazur games have natural generalizations to other lengths $\delta$ : At a limit stages $\gamma$, we use $\bigcap_{\alpha<\gamma} S_{\alpha}$ instead of $S_{\gamma-1}$, and empty wins a run iff this set is in $I$ for any $\gamma<\delta$. (I.e., nonempty wins a run iff the run has length $\delta$. So in this setting, the games defined above are the ones of length $\omega+1$.) For the $\neq \emptyset$ versions of the games, lengths other than $\omega+1$ seem less natural.

We are interested in the existence of winning strategies:

Definition 1.3. • We write $\mathfrak{b}(G)$ for "nonempty wins $G$ " and $\mathfrak{a}(G)$ for "empty does not win $G^{\prime \prime}$.

- The games $G$ and $H$ are equivalent, if $\mathfrak{b}(G) \leftrightarrow \mathfrak{b}(H)$ and $\mathfrak{a}(G) \leftrightarrow \mathfrak{a}(H)$.

- $G$ is stronger than $H$, if $\mathfrak{b}(G) \rightarrow \mathfrak{b}(H)$ and $\mathfrak{a}(G) \rightarrow \mathfrak{a}(H)$.

We trivially get the following implications, see Figure 1

Facts 1.4. $\quad \bullet \mathfrak{b}(G) \rightarrow \mathfrak{a}(G)$ for all games.

- The Banach-Mazur game is stronger than the according pressing down game. E.g., $\mathrm{BM}_{\mathrm{ne}}(I)$ is stronger than $\mathrm{PD}(I)$ etc.

- The $\notin I$ version is stronger than the $\neq \emptyset$ one. E.g., $\operatorname{BM}(I)$ is stronger than $\operatorname{Id}(I)$ etc. 


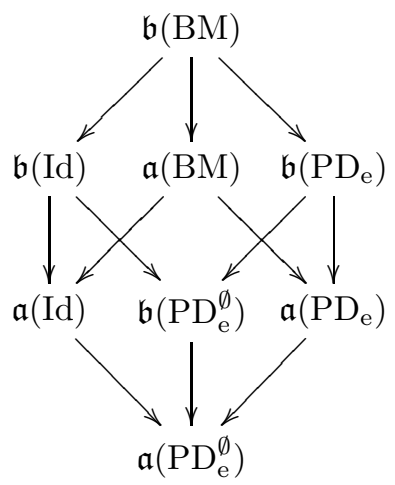

Figure 1. The trivial implications (for empty moving first)

- The version with empty choosing first is stronger. E.g., $\mathrm{BM}(I)$ is stronger than $\mathrm{BM}_{\mathrm{ne}}(I)$ etc.

We now list some well known (or otherwise obvious) facts about BM and precipitous idealı], see [11, 5, 9]:

Facts 1.5. - $\mathfrak{a}(\operatorname{Id}(I))$ is equivalent to " $I$ is precipitous".

- $\mathfrak{a}\left(\operatorname{Id}_{\text {ne }}(I)\right)$ is sometimes called " $I$ is somewhere precipitous", and its failure " $I$ is nowhere precipitous".

- A precipitous ideal on $\kappa$ implies that $\kappa$ is measurable in an inner model.

- $\mathfrak{b}\left(\mathrm{BM}\left(\mathrm{NS}_{\aleph_{2}}\left\lceil E_{\aleph_{1}}^{\aleph_{2}}\right)\right)\right.$ is equiconsistent to a measurable.

- "NS$\aleph_{\aleph_{1}}$ is precipitous" is also equiconsistent to a measurable.

- $\mathfrak{b}(\operatorname{Id}(I))$ implies $\kappa>2^{\aleph_{0}}$ and $E_{\aleph_{0}}^{\kappa} \in I$.

- $\mathfrak{a}(\operatorname{BM}(I))$ implies $E_{\aleph_{0}}^{\kappa} \in I$, and in particular $\kappa>\aleph_{1}$.

Some obvious facts about PD (for normal ideals $I, J$ ):

Facts 1.6. - In the pressing down games, we can assume without loss of generality that nonempty chooses at stage $n$ a set of the form $S_{n}=f_{n}^{-1}\left(\alpha_{n}\right) \cap$ $S_{n-1}$ for some $\alpha_{n} 2$

- $\mathrm{PD}$ is monotone in the following sense: if $J \supseteq I$, then $\operatorname{PD}(J)$ is stronger than $\mathrm{PD}(I)$. The same holds for $\mathrm{PD}^{\emptyset}$, but not for $\mathrm{PD}_{\mathrm{e}}$ nor $\mathrm{PD}_{\mathrm{e}}^{\emptyset}$ nor for any of the Banach Mazur games.

- In particular, $\mathrm{PD}(I)$ is stronger than $\mathrm{PD}\left(\mathrm{NS}_{\kappa}\right)$ for all normal $I$.

- Just as in the case of BM, $\mathfrak{b}\left(\mathrm{PD}^{\emptyset}\right)$ cannot hold for $\kappa=\aleph_{1}$ (cf. 5.2).

- Other than in the case of Id, the property $\mathfrak{a}\left(\mathrm{PD}_{\mathrm{e}}\right)$ has no consistency strength (cf. 2.1).

What is the effect of empty moving first?

Facts 1.7. - For the Banach-Mazur games, the distinction whether empty has the first move or nonempty is a simple density effect: For example, nonempty wins $\mathrm{BM}_{\text {ne }}(I)$ iff there is some $S \in I^{+}$such that nonempty

\footnotetext{
${ }^{1}$ these facts do not require that $I$ is normal

${ }^{2}$ This of course means: $\mathrm{PD}$ is equivalent to the game where nonempty is restricted to moves of this form.
} 
wins $\operatorname{BM}(I \uparrow S)$; similarly simple equivalences hold for empty winning; for characterizing $\mathrm{BM}$ in terms of $\mathrm{BM}_{\mathrm{ne}}$; and for the $\neq \emptyset$ version.

- We will see in Lemma 2.6 that this is not the case for the pressing down game.

The $\notin I$ versions of $\mathrm{BM}$ and PD are in fact instances of the cut and choose game introduced by Jech [12] (and its ancestor, the Ulam game):

Definition 1.8. The cut and choose game $\operatorname{c\& c}(B, \lambda)$ on a Boolean algebra $B$ is played as follows: First empty chooses a nonzero element $a_{0}$ of $B$. At stage $n$, empty chooses a maximal antichain $A_{n}$ below $a_{n}$ of size at most $\lambda$, and nonempty chooses an element $a_{n+1}$ from $A_{n}$. Nonempty wins the run if there is some nonzero $b$ below all $a_{n}$.

$c \& c(B, \infty)$ is played without restriction on the size of the antichains.

Let $\operatorname{ro}(B)$ denote the completion of the Boolean algebra $B$, and set $B_{I}=\mathfrak{P}(\kappa) / I$. The following can be found, e.g., in [13, 4, 19, 20]:

Facts 1.9. $\quad-\operatorname{c\& c}(B, \infty)$ is equivalent to $c \& c(\operatorname{ro}(B), \infty) 3$

- $\operatorname{c\& c}(B, \infty)$ is equivalent to the Banach Mazur game on $B$.

- In particular, $\mathrm{c} \& \mathrm{c}\left(B_{I}, \infty\right)$ is equivalent to $\operatorname{BM}(I)$.

- $\operatorname{c\& c}\left(B_{I}, \kappa\right)$ is equivalent to $\operatorname{PD}_{\mathrm{e}}(I)$, cf. 3.2 .

(However c\&c $\left(\operatorname{ro}\left(B_{I}\right), \kappa\right)$ might be a stronger game.)

It is less clear how the $\neq \emptyset$-versions of $\mathrm{BM}$ and $\mathrm{PD}$ relate to possible set-versions of the cut-and-choose game. On natural candidate is a "set-partition" game:

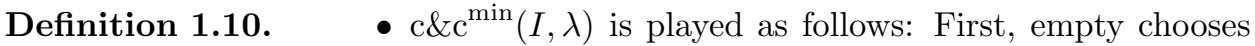
some positive $S_{-1}$. At stage $n$, empty partitions the set $S_{n-1}$ into at most $\lambda$ many (arbitrary) pieces, and nonempty chooses an $I$-positiv 4 piece $S_{n}$. Empty wins the run iff $\bigcap_{n \in \omega} S_{n}=\emptyset$.

- In $c \& \mathrm{c}^{\min }(I,<\kappa)$ empty cuts into less than $\kappa$ many (arbitrary) pieces.

- $c \& c_{n e}^{\min }$ is defined as usual, i.e., $S_{-1}=\kappa$

The following is straigthforward:

Fact 1.11. $\mathrm{PD}_{\mathrm{e}}^{\emptyset}(I)$ is stronger than $c \& c^{\min }(I,<\kappa)$, and $c \& c^{\min }(I,<\kappa)$ is stronger than $c \& c^{\min }(I, 2)$.

Remark 1.12. Another variant: Empty has to partition into positive pieces (of size at most $\lambda$ ), and wins a run iff the intersection is in $I$. Let us call this game $c \& c^{\text {set }}(I, \lambda)$ (we will not need it in the rest of the paper). It is not entirely clear how this game relates to the previous ones:

Obviously there can be at most $\kappa$ many pieces, so $c \& c^{\mathrm{set}}(I, \infty)=\mathrm{c} \& \mathrm{c}^{\mathrm{set}}(I, \kappa)$.

For $\lambda<\kappa$ it is easy to see that $c \& c^{\text {set }}(I, \lambda)$ is equivalent to $c \& c\left(B_{I}, \lambda\right)$.

Also, it is clear that $c \& c^{\text {set }}(I, \kappa)$ is stronger than $c \& c\left(B_{I}, \kappa\right)$, which is equivalent to $\operatorname{PD}_{\mathrm{e}}(I)$.

The relation of ${\mathrm{c} \& \mathrm{c}^{\mathrm{set}}}^{\mathrm{s}}(I, \kappa)$ and $\mathrm{BM}(I)$ is less clear. Of course, if $I$ is $\kappa^{+}$-saturated, then $c \& c^{\text {set }}(I, \kappa), c \& c\left(B_{I}, \kappa\right), \mathrm{PD}_{\mathrm{e}}(I)$ and $\mathrm{BM}(I)$ are all equivalent, $\mathrm{cf}$. 3.2 and 3.3 .

\footnotetext{
${ }^{3} \operatorname{But} \mathrm{c} \& \mathrm{c}(B, \lambda)$ will generally not be equivalent to $\mathrm{c} \& \mathrm{c}(\operatorname{ro}(B), \lambda)$.

${ }^{4}$ Note that we allow empty to include $I$-null pieces into the partition, but we require nonempty to choose a positive piece; otherwise nonempty always wins by picking right from the start an element $\alpha$ and then always picking the piece containing $\alpha$.
} 


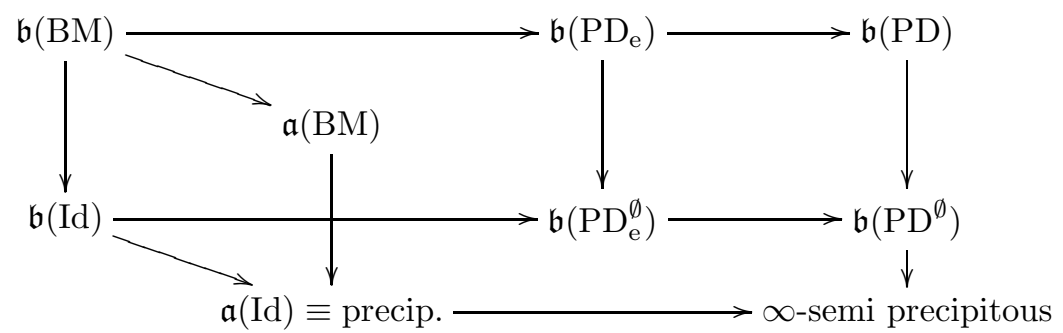

Figure 2. Some properties stronger than $\infty$-semi precipitous

Winning strategies for games on a Boolean algebra $B$ have close connections to the properties of $B$ as Boolean algebra and as forcing notions, again see [13, 4, 19, 20]:

Facts 1.13. $\quad-B$ having a $\sigma$-closed positive subset implies $\mathfrak{b}(\operatorname{BM}(B))$.

- $\mathfrak{b}(\operatorname{BM}(B))$ is also denoted by " $B$ is strategically $\sigma$-closed" and implies that $B$ is proper.

- $\mathfrak{a}(\operatorname{BM}(B))$ is equivalent to " $B$ is $\sigma$-distributive".

It is not surprising that we will get stronger connections if we assume that the $B$ has the form $B_{I}=\mathfrak{P}(\kappa) / I$ for a normal ideal $I$. We will mention only one example:

Fact 1.14. If $B_{I}$ is proper and $\kappa>2^{\aleph_{0}}$, then $\mathfrak{a}(\operatorname{BM}(I))$ holds.

For a proof, see 3.5 .

\section{THE RESUlts}

Some of the facts for precipitous ideals can be shown (with similar proofs) for PD, but there are of course strong differences as well:

Lemma 2.1. $\quad(1) \mathfrak{b}\left(c \mathscr{G} c_{n e}^{\text {min }}(I, 2)\right)$ implies that $\kappa$ is measurable in an inner model.

(2) So in particular, $\mathfrak{b}\left(P D^{\emptyset}(I)\right)$ implies that as well.

(3) However, $\mathfrak{a}\left(P D_{e}(I)\right)$ has no consistency strength. In particular, for $\kappa=\aleph_{2}$, $\mathfrak{a}\left(P D_{e}(I)\right)$ is implied by $C H$ for every $I$ concentrated on $E_{\aleph_{1}}^{\aleph_{2}}$.

(4) $\mathfrak{b}\left(P D^{\emptyset}(I)\right)$ implies $\kappa>2^{\aleph_{0}}$ and that $I$ is not concentrated on $E_{\aleph_{0}}^{\kappa}$.

The proofs can be found in 5.5, 5.2 and 5.3.

In this paper, we are not interested in the property "empty does not win the pressing down game", since it has no consistency strength. Also, the effect of who moves first in Banach Mazur games is trivial. The remaining properties are pictured in Figure 2. All these properties are equiconsistent to a measurable (e.g., for $I=$ $\left.\mathrm{NS}_{\aleph_{2}} \uparrow E_{\aleph_{1}}^{\aleph_{2}}\right)$. In fact, they imply that $I$ is $\infty$-semi precipitous, see Definition 4.1, which in turn implies that $\kappa$ is measurable in an inner model. We claim that none of the implications can be reversed. In this paper, we will prove some strong instances of this claim by assuming larger cardinals: We show

- $\mathfrak{b}\left(\mathrm{PD}_{\mathrm{e}}\right)$ does not imply precipitous, and

- $\mathfrak{a}(\mathrm{BM})$ does not imply $\mathfrak{b}\left(\mathrm{PD}^{\emptyset}\right)$.

We also claim that (consistently relative to a measurable)

- $\mathfrak{b}(\mathrm{Id})$ does not imply $\mathfrak{b}(\mathrm{PD})$, 
but we do not give a proof here. With these claims (for which we assume cardinals larger than a measurable) it is then easy to check that no implication of Figure 2 can be reversed.

In [15, Pauna and the authors showed that, assuming the consistency of a measurable, $\mathfrak{b}(\mathrm{PD}(I))$ does not imply $\mathfrak{b}\left(\mathrm{BM}_{\mathrm{ne}}(I)\right)$ for $I=\mathrm{NS}_{\aleph_{2}}\left\lceil E_{\aleph_{1}}^{\aleph_{2}}\right.$. In fact, a slightly stronger statement holds (with a simpler proof):

Lemma 2.2. It is equiconsistent with a measurable that $\mathfrak{b}(P D(I))$ holds (even for length $\left.\omega_{1}\right)$ but $\left.\mathfrak{a}\left(I_{n e}(I)\right)\right)$ fails for $I=\mathrm{NS}_{\aleph_{2}}\left\lceil E_{\aleph_{1}}^{\aleph_{2}}\right.$.

(For a proof, see [5.8.) Note that " $\left.\mathfrak{a}\left(\operatorname{Id}_{\text {ne }}(I)\right)\right)$ fails" just means that $I$ is nowhere precipitous.

Of course, precipitous cannot generally imply a winning strategy for nonempty in any game, since precipitousness is consistent with $\kappa \leq 2^{\aleph_{0}}$. However, we can get counterexamples for $\kappa>2^{\aleph_{0}}$ as well: Just adding Cohens destroys any winning strategy for nonempty (for any ideal on $\aleph_{2}$ ), but preserves precipitous. So we get (see 5.10 and 5.11):

Lemma 2.3. It is equiconsistent with a measurable that $C H$ holds, $\left.\mathrm{NS}_{\aleph_{2}}\right\rceil E_{\aleph_{1}}^{\aleph_{2}}$ is precipitous but $\mathfrak{b}\left(P D^{\emptyset}(J)\right)$ fails for any normal ideals $J$ on $\aleph_{2}$.

To see that not even $\mathfrak{a}(\operatorname{BM}(I))$ implies any winning strategy for nonempty, we assume $\mathrm{CH}$ and a $\aleph_{3}$-saturated ideal $I$ on $\aleph_{2}$ concentrated on $E_{\aleph_{1}}^{\aleph_{2}}$. Saturation is preserved by small forcings, in particular by adding some Cohens, and saturation (together with $\mathrm{CH})$ implies $\mathfrak{a}(\mathrm{BM}(I))$. So we get:

Lemma 2.4. The following is consistent with $C H$ plus an $\aleph_{3}$-saturated ideal on $\aleph_{2}$ : $C H$ holds, $\mathfrak{a}(B M(I))$ holds for some I on $\aleph_{2}$, but $\mathfrak{b}\left(P D^{\emptyset}(J)\right)$ fails for any normal $J$ on $\aleph_{2}$.

See 5.12. (It seems very likely that saturation is not needed for this, but the construction might get considerably more complicated without it.)

As mentioned in Lemma 2.2 it is possible that $\mathfrak{b}(\mathrm{PD}(I))$ holds for a nowhere precipitous ideal, i.e., for an ideal such that $\mathfrak{a}\left(\operatorname{Id}_{\text {ne }}(I)\right)$ fails. With a bit more work, we even get $\mathfrak{b}\left(\mathrm{PD}_{\mathrm{e}}(I)\right)$ :

Theorem 2.5. It is equiconsistent with a measurable that for $\kappa=\aleph_{2}$ there is a nowhere precipitous $I$ such that $\mathfrak{b}\left(P D_{e}(I)\right)$ holds (even for length $\left.\omega_{1}\right)$.

(See Fact 6.1)

Note that (as opposed to 2.3, 2.4) we just make a specific ideal non-precipitous, and we do not destroy all precipitous ideals. It seems very hard (and maybe impossible) to do better: It is not known how to kill all precipitous ideal.5 on, e.g., $\aleph_{1}$ with "reasonable" forcings 9 And it might be even harder to do so while additionally preserving $\mathfrak{b}\left(\mathrm{PD}_{\mathrm{e}}(I)\right)$ for some ideals: By recent results by Gitik 7] (and later Ferber and Gitik [3] ) a $\infty$-semi precipitous ideal does imply a normal precipitous ideal under in the absence of larger cardinals and under some cardinal arithmetic assumptions.

\footnotetext{
${ }^{5}$ Since we are only interested in normal ideals, it would be enough to kill all normal precipitous ideals. This doesn't help much, though; it is not known whether the existence of a precipitous ideal does imply the existence of a normal precipitous one. Recently Gitik [6, 7, 3] proved some interesting results in this direction.

${ }^{6}$ More specifically, it is not known whether large cardinals imply a precipitous ideal on $\aleph_{1}$, although Woodins are not enough, cf. [18].
} 
2.1. Moving first. Let us now investigate the effect of whether empty moves first.

If we compare $G_{\mathrm{e}}$ and $H_{\mathrm{ne}}$ for any games $G$ and $H$, then these variants will be different for trivial reasons: For example, $\mathfrak{b}\left(\mathrm{BM}_{\text {ne }}(I)\right)$ does not imply $\mathfrak{b}\left(\mathrm{PD}_{\mathrm{e}}^{\emptyset}(I)\right)$ : Let $U$ be a normal ultrafilter on $\kappa$, Levy-collapse $\kappa$ to $\aleph_{2}$, and let $I_{1}$ be the ideal generated by the dual of $U$ (which is concentrated on $E_{\aleph_{1}}^{\aleph_{2}}$ ). Then nonempty wins $\operatorname{BM}\left(I_{1}\right)$ and therefore $\operatorname{BM}_{n e}(I)$ as well for $\left.I=I_{1}+\mathrm{NS}_{\aleph_{2}} \uparrow E_{\aleph_{0}}^{\aleph_{2}}\right)$ as well. But nonempty can never win $\mathrm{PD}_{\mathrm{e}}^{\emptyset}(I)$, since nonempty cannot win $\mathrm{PD}^{\emptyset}\left(\mathrm{NS}_{\aleph_{2}}\left\lceil E_{\aleph_{0}}^{\aleph_{2}}\right)\right.$. The same holds for $I=\mathrm{NS}_{\aleph_{2}}$ (just use the model of $\mathfrak{b}\left(\mathrm{BM}\left(\mathrm{NS}_{\aleph_{2}}\left\lceil E_{\aleph_{1}}^{\aleph_{2}}\right)\right.\right.$ ).

So the games are very different (for trivial reasons) when we change who has the first move. However, for the Banach Mazur game, the effect of who moves first is a simple density effect 7 as we have mentioned in 1.7. For example, $\mathfrak{b}\left(\mathrm{BM}_{\mathrm{ne}}(I)\right)$ holds iff $\mathfrak{b}(\operatorname{BM}(I \uparrow S))$ holds for some positive $S$.

This is not the case for the pressing down games. Of course we still get:

- $\mathfrak{b}\left(\mathrm{PD}_{\mathrm{e}}(I)\right)$ holds iff $\mathfrak{b}(\mathrm{PD}(I \uparrow S))$ holds for all $S \in I^{+}$.

- The same holds for $\mathrm{PD}_{\mathrm{e}}^{\emptyset}$.

But unlike the Banach Mazur case, we can have the following:

Lemma 2.6. It is equiconsistent with a measurable that $\mathfrak{b}(P D(I))$ holds but $\mathfrak{b}\left(P D_{e}(I \mid\right.$ $S$ )) fails for all positive $S$, e.g., for $I=\mathrm{NS}_{\aleph_{2}}$.

(See 5.8) So in other words, $\mathfrak{b}(\mathrm{PD}(I))$ can hold but for all positive $S$ there is a positive $S^{\prime} \subseteq S$ such that $\mathfrak{b}\left(\operatorname{PD}\left(I \uparrow S^{\prime}\right)\right)$ fails.

\section{EMPTY NOT WINNING}

Lemma 3.1. - $\quad C H$ implies $\mathfrak{a}(P D(I))$ for every $I$ on $\aleph_{2}$ that is not concentrated on $E_{\aleph_{0}}^{\aleph_{2}}$.

- More generally, if $\lambda^{\aleph_{0}}<\kappa$ for all $\lambda<\kappa$, then empty wins $P D(I)$ iff $E_{>\omega}^{\kappa} \in$ $I$.

- So if $I$ is concentrated on $E_{>\omega}^{\kappa}$ (and the same cardinal assumptions hold) then $\mathfrak{a}\left(P D_{e}(I)\right)$ holds.

Proof. Assume that $I$ is concentrated on $E_{\aleph_{0}}^{\kappa}$. Just as in [5], it is easy to see that empty wins $\operatorname{PD}(I)$ : For every $\alpha \in E_{\aleph_{0}}^{\kappa}$, let $(\operatorname{seq}(\alpha, n))_{n \in \omega}$ be a cofinal sequence in $\alpha$. Let $F_{n}$ map $\alpha$ to seq $(\alpha, n)$. If empty plays $F_{n}$ at stage $n$, then the intersection can contain at most one element.

So assume towards a contradiction that $E_{>\omega}^{\kappa} \notin I$ and that empty has a winning strategy for $\operatorname{PD}(I)$. The strategy assigns sets $X_{t}$ and regressive function $f_{t}$ to nodes $t$ in the tree $T=\kappa^{<\omega}$ in the following way:

For $t=\langle\rangle$, set $X_{\langle\rangle}=\kappa$ and let $f_{\langle\rangle}$be empty's first move. For $\alpha \in \kappa$, set $X_{(\alpha)}=f_{t}^{-1}(\alpha)$. Note that $\alpha$ is a valid response for nonempty iff $X_{(\alpha)}$ is positive. Generally, fix $t \in T$. We can assume by induction that one of the following cases hold:

- $t$ corresponds to a partial run $r_{t}$ with (positive) partial result $X_{t}$; then we set $f_{t}$ to be empty's response to $r_{t}$

- $X_{t} \in I$; then we set $f_{t} \equiv 0$.

\footnotetext{
${ }^{7}$ In games of length bigger than $\omega+1$ however it does make a substantial difference who moves first at limits.
} 
In both cases we set $X_{t-\alpha}=X_{t} \cap f_{t}^{-1}(\alpha)$.

Let $b$ be a branch of $T$ (i.e., $b \in \kappa^{\omega}$ ). We set $X^{b}=\bigcap_{n \in \omega} X_{b\lceil n}$.

Assume that $b$ corresponds to a run of the game; this is the case iff $X_{b \nmid n}$ is $I$-positive for all $n$. Then $X^{b} \in I$, since empty uses the winning strategy. If $b$ does not correspond to a run, then $X^{b} \in I$ as well. So

$$
X^{b} \in I \text { for all branches } b
$$

$X^{b}$ and $X^{c}$ are disjoint for different branches $b, c$; and for all $\gamma \in \kappa$ there is exactly one branch $b_{\gamma}$ such that $\gamma \in X^{b_{\gamma}}$. We assume $\gamma \neq 0$ from now on. By definition, for all $n$

$$
f_{b_{\gamma}\lceil n}(\gamma)=b_{\gamma}(n)
$$

Since $f_{b_{\gamma} \mid n}$ is regressive, $b_{\gamma}(n)<\gamma$ for all $n \in \omega$. In other words, $b_{\gamma} \in \gamma^{\omega}$.

Fix an injective function $\phi: \kappa^{\omega} \rightarrow \kappa$. Since $\gamma^{\aleph_{0}}<\kappa$ for $\gamma<\kappa$, we can find a club $C$ such that

$$
\phi^{\prime \prime} \gamma^{\omega} \subseteq \gamma \text { for all } \gamma \in C \cap E_{>\omega}^{\kappa} .
$$

This defines a regressive function $g: C \cap E_{>\omega}^{\kappa} \rightarrow \kappa$ by $g(\gamma)=\phi\left(b_{\gamma}\right)$. Since $I$ is normal and does not contain $E_{>\omega}^{\kappa}$, there is a positive set $S$ and a $\zeta \in \kappa$ (or equivalently a branch $b$ of $T$ ) such that $g(\gamma)=\zeta$, i.e., $b_{\gamma}=b$ for all $\gamma \in S$. This implies that $S \subseteq X^{b}$ is positive, a contradiction to (1).

Lemma 3.2. If $I$ is normal, then $P D_{e}$ is equivalent to $c \mathscr{B} c\left(B_{I}, \kappa\right)$.

Proof. A regressive function defines a maximal antichain in $B_{I}$ of size at most $\kappa$. On the other hand, let $A$ be a maximal antichain of size $\lambda \leq \kappa$. We can choose pairwise disjoint representatives $\left(S_{i}\right)_{i \in \lambda}$ for the elements of $A$, and define

$$
f(\alpha)= \begin{cases}1+i & \text { if } \alpha \in S_{i} \text { and } 1+i<\alpha, \\ 0 & \text { otherwise. }\end{cases}
$$

$f^{-1}(0) \in I$. (Otherwise there is an $S_{i}$ in $A$ such that $T=S_{i} \cap f^{-1}(0) \in I^{+}$, pick $\alpha \in T \backslash(1+i+1)$, contradiction.) So the partition $A$ is equivalent to the regressive function $f$.

Together with 1.9 we get:

Corollary 3.3. If $I$ is normal and $\kappa^{+}$-saturated, then $B M(I)$ and $P D_{e}(I)$ are equivalent. The same holds for $B M_{n e}(I)$ and $P D(I)$.

If $I$ is $\kappa^{+}$-saturated, then it is precipitous, i.e., $\mathfrak{a}(\operatorname{Id}(I))$ holds [10, 22.22]. However, $I$ can be concentrated on $E_{\aleph_{0}}^{\kappa}$ (for example, $\kappa$ could be $\aleph_{1}$ ), which negates $\mathfrak{a}(\operatorname{BM}(I))$. However, with Lemma 3.1 we get:

Corollary 3.4. If $I$ is $\kappa^{+}$-saturated, $\lambda^{\aleph_{0}}<\kappa$ for all $\lambda<\kappa$, and $I$ is normal and concentrated on $E_{>\aleph_{0}}^{\kappa}$, then $\mathfrak{a}(B M(I))$ holds.

In the rest of the section, we show that properness implies $\mathfrak{a}(\operatorname{BM}(I))$. This is not needed for the rest of the paper.

For any Boolean algebra $B, \mathfrak{b}(\operatorname{BM}(B))$ implies that $B$ is proper (as a forcing notion), cf. e.g. [12, Thm. 7]. For Boolean algebras of the form $B_{I}=\mathfrak{P}(\kappa) / I$ we also get:

Lemma 3.5. Assume $\kappa>2^{\aleph_{0}}$. If $B_{I}$ is proper then $\mathfrak{a}(B M(I))$ holds.

Normality of $I$ is not needed, just $<\kappa$-completeness. 
Proof. Assume towards a contradiction that $\tau$ is a winning strategy for empty. Let $p_{0} \in I^{+}$be empty's first move according to $\tau$. Pick $N \prec H(\chi)$ countable containing $I$ and $\tau$ (and therefore $p_{0}$ ), and let $q \leq p_{0}$ be $N$-generic. In other words, if $\mathcal{D} \in N$ is a predense subset of $I^{+}$, then $q$ is $(\bmod I)$ a subset of $\bigcup(\mathcal{D} \cap N)$. Therefore

$$
\mathcal{X}=q \cap \bigcap\left\{\bigcup(\mathcal{D} \cap N): \mathcal{D} \subseteq I^{+} \text {is predense and } \mathcal{D} \in N\right\},
$$

is positive. We set

$$
\mathcal{Y}=\bigcup\left\{\bigcap_{n \in \omega} A_{n}:(\forall n \in \omega) A_{n} \in N \cap I^{+}, \bigcap_{n \in \omega} A_{n} \in I\right\}
$$

$\mathcal{Y} \in I$, since $\left|[N]^{\aleph_{0}}\right|<\kappa$. So we can pick some

$$
\delta^{*} \in \mathcal{X} \backslash \mathcal{Y} \text {. }
$$

We now construct a run of the game such that every initial segment is in $N$. Assume that we already know the initial segment of the first $n-1$ stages, and that this segment is in $N$. Then empty's move $A_{n}$ given by $\tau$ is in $N$ as well. We further assume that $\delta^{*} \in A_{n}$. (This is true for $n=0$, since $\delta^{*} \in q \leq p_{0}$.) For any $I$-positive $B \subseteq A_{n}$ let empty's response be $f(B)$. The set

$$
D=\left\{\kappa \backslash A_{n}\right\} \cup\left\{f(B): B \subseteq A_{n} \text { positive }\right\}
$$

is dense in $I^{+}$and is in $N$. Since $\delta^{*} \in \mathcal{X}, \delta^{*} \in \bigcup(D \cap N)$, i.e. there is some $B \in N$ such that $\delta^{*} \in f(B)$. Let $B$ be nonempty's move.

So $\delta^{*}$ will be in the intersection $Z=\bigcap_{n \in \omega} A_{n}$, and since empty wins the run, $Z \in I$. Since each $A_{n}$ is in $N$, we get $Z \subseteq \mathcal{Y}$. This contradicts $\delta^{*} \in Z$.

\section{4. $\infty$-SEMI PRECIPITOUS IDEALS}

Definition 4.1. A $\kappa$-complete ideal $I$ on $\kappa$ is called (normally) $\infty$-semi precipitous, if there is some partial order $P$ which forces that there is a (normal) wellfounded, nonprincipal, $\kappa$-complete $V$-ultrafilter containing the dual of $I$.

Donder, Levinski [2] introduced the notion of $\lambda$-semi precipitous, and Ferber and Gitik [3] extended the notation to $\infty$-semi precipitous. Another name, "weakly precipitous", is used for this notion in 11. However, Jech uses the term "weakly precipitous" for another concept, cf. [13, 2].

We will see in Lemma 5.5 that $\mathfrak{b}\left(\operatorname{PD}^{\emptyset}(I)\right)$ implies that $I$ is normally $\infty$-semi precipitous. This will establish the consistency strength of $\mathfrak{b}\left(\mathrm{PD}^{\emptyset}(I)\right)$ :

Lemma 4.2. If there is an $\infty$-semi precipitous ideal on $\kappa$, then $\kappa$ is measurable in an inner model.

This is of course no surprise: the proof is a simple generalization of the proof 9 , Theorem 2] for precipitous; Jech and others have used in fact very similar generalizations. (E.g., in [13] it is shown more or less that pseudo-precipitous ideals are $\infty$-semi precipitous.)

Proof. We assume that there is a forcing $P$ and a name $\underset{\sim}{D}$ for the $V$-generic filter. In particular:

$P$ forces that in $V[G]$ there is an elementary embedding $j: V \rightarrow M$ for some transitive class $M$ in $V[G]$.

If we are only interested in consistency strength, we can use Dodd-Jensen core model theory as a black-box: (2) is equiconsistent to a measurable cardinal, which 
follows immediately, e.g., from [10, 35.6] and the remark after [10, 35.14]: $K^{V}=$ $K^{V[G]}$, and there is a measurable iff there is an elementary embedding $j: K \rightarrow M$ (which also implies $M=K$ ). However, this only tells us that there is some ordinal which is measurable in an inner model, and not that this ordinal is indeed $\kappa$.

To see this, we can either use more elaborate core model theory (as pointed out by Gitik, cf. [21, 7.4.8,7.4.11]). Alternatively, we can just slightly modify the proof of [9, Theorem 2] (which can also be found in [10, 22.33]). We will do that in the following: Let $K$ be the class of strong limit cardinals $\mu$ such that $\operatorname{cf}(\mu)>\kappa$ and $\mu>|P|$. Let $\left(\gamma_{n}\right)_{n \in \omega}$ be an increasing sequence in $K$ such that $\left|K \cap \gamma_{n}\right|=\gamma_{n}$. Set $A=\left\{\gamma_{n}: n \in \omega\right\}$ and $\lambda=\sup (A)$.

By a result of Kunen, it is enough to show the following:

There is (in $V$ ) an iterable, normal, fine $L[A]$-ultrafilter $W$ such that every iterated ultrapower is wellfounded.

We have a name $\underset{\sim}{D}$ for the $V$-generic filter. $\underset{\sim}{D}$ does not have to be normal, but there is some $p_{0} \in P$ and $\alpha_{0} \geq \kappa$ such that $p_{0}$ forces that $[\mathrm{Id}]=\alpha_{0}$. We set

$$
\begin{gathered}
\mathcal{J}=\left\{x \subseteq \kappa: p_{0} \Vdash x \notin \underset{\sim}{D}\right\}, \text { and } \\
U=\{x \in \mathfrak{P}(\kappa) \cap L[A]: x \notin \mathcal{J}\} .
\end{gathered}
$$

$U$ is generally not normal, but the normalized version of $U$ will be as required.

$U$ is an $L[A]$ ultrafilter: Let $x \subseteq \kappa$ be in $L[A]$. We have to show: $x$ or $\kappa \backslash x$ are in $\mathcal{J}$.

- There is a formula $\varphi$ and a finite $E \subseteq \kappa \cup K$ such that (in $L[A]) \alpha \in x$ iff $\alpha<\kappa$ and $\varphi(\alpha, E, A)$.

- Assume $G$ is $P$-generic over $V$ and contains $p_{0}$. [Id] $=\alpha_{0}$, so $x \in \underset{\sim}{D}[G]$ iff $\alpha_{0} \in j(x)$.

- By elementarity (in $V[G]) \alpha_{0} \in j(x)$ iff $j(L[A])$ thinks that $\varphi\left(\alpha_{0}, j(E), j(A)\right.$ ). But $j(\mu)=\mu$ for every $\mu \in K$.

- So we get $x \in \underset{\sim}{D}[G]$ iff (in $L[A]) \varphi\left(\alpha_{0}, E, A\right)$ holds, independently of $G$ (provided $G$ contains $p_{0}$ ). In other words, if there is some generic $G$ such that $x \in \underset{\sim}{D}[G]$, then $x \in \underset{\sim}{D}[G]$ for all generic $G$ (containing $p_{0}$ ); i.e. $p_{0}$ forces that $x \in \underset{\sim}{D}[G]$; i.e. $\kappa \backslash x \in \mathcal{J}$.

- Assume that $x$ is not in $\mathcal{J}$. Then there is some $q \leq p_{0}$ forcing that $x \in D$. So $\kappa \backslash x \in \mathcal{J}$.

$U$ is $<\kappa$-complete, fine and wellfounded: Pick $\lambda<\kappa$ and $\left(x_{\alpha}\right)_{\alpha \in \lambda}$ in $L[A]$ such that each $x_{\alpha} \in U$. Then $p_{0}$ forces that $\kappa \backslash x_{\alpha} \notin \underset{\sim}{D}$, and therefore that $\bigcup \kappa \backslash x_{\alpha} \notin \underset{\sim}{D}$ (since $\underset{\sim}{D}$ is a $<\kappa$-complete ultrafilter).

This also shows that (in $V$ ) the intersection of $\aleph_{0}$ many $U$-elements is nonempty; which implies that every iterated ultrapower is wellfounded (provided iterability).

$U$ is iterable: Let (in $L[A])\left(x_{\alpha}\right)_{\alpha \in \kappa}$ be a sequence of subsets of $\kappa$. Let $G$ be $P$-generic over $V$ and contain $p_{0}$. In $V[G], x_{\alpha} \in D[G]$ iff $\alpha_{0} \in j\left(x_{\alpha}\right)$. The sequence $\left(j\left(x_{\alpha}\right)\right)_{\alpha \in \kappa}$ is in $L[j(A)]$, and therefore also the set $\left\{\alpha \in \kappa: \alpha_{0} \in j_{G}\left(x_{\alpha}\right)\right\}$. But $L[j(A)]=L[A]$.

normalizing: Since we now know that $U$ is wellfounded, we know that there is some $f: \kappa \rightarrow \kappa$ in $L[A]$ representing $\kappa$. Set $W=f_{*}(U)$. Then $W$ is as required.

The following follows easily from Kunen's method of iterated ultrapowers (see, e.g., [15, 4.3] for a proof): 
Lemma 4.3. Assume $V=L[U]$, where $U$ is a normal ultrafilter on $\kappa$. Let $V^{\prime}$ be a forcing extension of $V$ and $D \in V^{\prime}$ a normal, wellfounded $V$-ultrafilter on $\kappa$. Then $D=U$.

This implies:

Corollary 4.4. In $L[U]$, the dual of $U$ is the only normal precipitous ideal on $\kappa$; and every ideal on $\kappa$ that is normally $\infty$-semi precipitous is a subideal of the dual of $U$.

We will also need the following:

Lemma 4.5. If $I$ is a $<\kappa$-complete ideal, $P$ a $\kappa$-cc forcing notion, and $\operatorname{cl}(I)$ the $P$-name for the closure of $I$ in $V[G]$, then $P$ preserves the following properties: $I$ is precipitous, $I$ is not precipitous, and $I$ is nowhere precipitous.

Proof. This has been known for a long time, cf. e.g. [14]: "not precipitous" is equivalent to the existence of a decreasing sequence of functionals starting at some positive set $S_{0}$ (this corresponds to: $S_{0}$ forces that there is an infinite decreasing sequence in the ultrapower, the sequence of functionals witnesses this). A $\kappa$-cc forcing preserves maximality (below $S_{0}$ ) of an antichain in $B_{I}$, and therefore the decreasing sequence of functionals. "Nowhere precipitous" is equivalent to the existence of a decreasing sequence of functionals starting with $\kappa$, which again is preserved by $P$.

\section{NONEMPTY WINNING}

Let us assume that nonempty has a winning strategy in $\mathrm{PD}^{\emptyset}(I)$ (or a similar game such as $\mathrm{PD}(I)$ ). A valid sequence is a finite initial sequence of a run of the game $\mathrm{PD}^{\emptyset}$, where nonempty uses his strategy. So a valid sequence $w$ has the form $\left(f_{0}, \alpha_{0}, f_{1}, \alpha_{1}, \ldots, f_{n-1}, \alpha_{n-1}\right)$, where $f_{i}$ is a regressive function and $\alpha_{i}$ the value chosen by the strategy. In particular $S_{i}=\bigcap_{j \leq i} f_{j}^{-1}\left(\alpha_{j}\right)$ is $I$-positive for each $i<n$. We set

$$
A(w)=S_{n-1}=\bigcap_{j<n} f_{j}^{-1}\left(\alpha_{j}\right) .
$$

Definition 5.1. $P^{*}$ is the set of valid sequences ordered by extension. (A longer sequence is stronger, i.e., smaller in the $P^{*}$-order.)

So if $w<v$, then $A(w) \subseteq A(v)$. If $w_{0}>w_{1}>w_{2}>\ldots$ is an infinite decreasing sequence in $P^{*}$, then $\bigcup_{i \in \omega} w_{i}$ represents a run of the game, so the result $\bigcap_{i \in \omega} A\left(w_{i}\right)$ has to be nonempty (or even positive in the case of a PD-strategy).

Lemma 5.2. $\mathfrak{b}\left(P D^{\emptyset}\right)$ implies $\kappa>2^{\aleph_{0}}$.

Actually, we can even restrict nonempty to play functions $f: \kappa \rightarrow\{0,1\}$. In other words, it is enough to assume $\mathfrak{b}\left(c \& c^{\min }(I, 2)\right)$, cf. Definition 1.10,

Proof. The proof is the same as [5, §1]: We assume otherwise and identify $\kappa$ with a subset $X$ of $[0,1]$ without a perfect subset. We claim:

For all $w \in P^{*}$ and $n \in \omega$ there are disjoint open intervals $I_{1}$ and $I_{2}$ of length $\leq 1 / n$ and $w_{1}, w_{2}<w$ such that $A\left(w_{1}\right) \subseteq I_{1}$ and $A\left(w_{2}\right) \subseteq I_{2}$. 
Assume that (4) fails for some $v_{0}$ and $n_{0}$. Given $v<v_{0}$ and $n>n_{0}$, we fix a partition of $[0,1]$ into $n$ many open intervals of length $1 / n$ and the (finite) set of endpoints. By splitting $A(v) n+1$ many times, empty can guarantee that $A(w)$ has to be subset of one of the intervals for some $w<v$. Since (44) fails, there has to be for each $n$ a fixed element $I(n)$ of the partition such that for all $v<v_{0}$ there is a $w<v$ with $A(w) \subseteq I(n)$. $\bigcap I(n)$ can contain at most one point $x$, so the empty player can continue $v_{0}$ by first splitting into $\{x\}$ and $A\left(v_{0}\right) \backslash\{x\}$; and then extending each $v_{n-1}$ to $v_{n}$ such that $A\left(v_{n}\right) \subseteq I(n)$. Then the intersection is empty. This shows (4).

So we can fix an order preserving function $\psi$ from $2^{<\omega}$ to $P^{*}$ such that $A(\psi(s \frown 0))$ and $A\left(\psi\left(s^{\frown} 1\right)\right)$ are separated by intervals of length $\leq 1 /|s|$ for all $s \in 2^{<\omega}$. Then every $\eta \in 2^{\omega}$ is mapped to a run of the game, and since nonempty wins, there is some $r_{\eta} \in \bigcap_{n \in \omega} A\left(\psi(\eta\lceil n))\right.$. This defines a continuous, injective mapping from $2^{\omega}$ into $X$ and therefore a perfect subset of $X$.

Clearly $\mathfrak{a}(\mathrm{PD})$ fails if $I$ is concentrated on $E_{\aleph_{0}}^{\kappa}$, and this was used in [5] to show that in this case $\mathfrak{b}\left(\operatorname{Id}_{\mathrm{ne}}(I)\right)$ fails as well. A similarly easy proof gives:

Lemma 5.3. $\mathfrak{b}\left(P D^{\emptyset}(I)\right)$ fails if $I$ is concentrated on $E_{\aleph_{0}}^{\kappa}$.

Proof. Assume otherwise. Fix for each $\alpha \in E_{\aleph_{0}}^{\kappa}$ a normal cofinal sequence $(\operatorname{seq}(\alpha, n))_{n \in \omega}$, and let $g_{i}: \kappa \rightarrow \kappa$ map $\alpha$ to $\operatorname{seq}(\alpha, i)$. We first show a variant of (4):

For all $w$ there are $v_{1}, v_{2} \leq w$ in $P^{*}$ such that $A\left(v_{1}\right) \cap A\left(v_{2}\right)=\emptyset$.

Assume otherwise. Then for each $i$ there is a fixed $\beta_{i}$ such that nonempty responds with $\beta_{i}$ whenever empty plays $g_{i}$ in any $v \leq w$. Set $\delta=\sup \left\{\beta_{i}: i \in \omega\right\}$, and let empty play the following response to $w$ :

$$
f(\alpha)= \begin{cases}0 & \text { if } \alpha \leq \delta \\ \min \{n: \operatorname{seq}(\alpha, n)>\delta\} & \text { otherwise }\end{cases}
$$

If nonempty responds to $f$ with $m$, then empty can play $g_{m}$ as next move, nonempty has to respond with $\beta_{m}<\delta$, but

$$
g_{m}^{-1}\left(\beta_{m}\right)=\left\{\alpha: \operatorname{seq}(\alpha, m)=\beta_{m}\right\}
$$

is disjoint to $f^{-1}(m)$, a contradiction. This shows (5).

Now fix $N \prec H(\chi)$ of size less than $\kappa$ containing the strategy as well as all $g_{n}$ and such that $N \cap \kappa=\delta \in E_{\aleph_{0}}^{\kappa}$. We define a sequence $w_{0}>w_{1}>\cdots$ in $P^{*}$ such that each $w_{i}$ is in $N$ : Using (5) in $N$, we get a $w_{0} \in N \cap P^{*}$ such that $\delta \notin A\left(w_{0}\right)$. Given $w_{n-1}$, let $w_{n} \in N$ be the continuation where empty played the regressive function

$$
f_{n}(\alpha)= \begin{cases}0 & \text { if } \alpha<\operatorname{seq}(\delta, n) \\ g_{n}(\alpha) & \text { otherwise }\end{cases}
$$

(Note that $\operatorname{seq}(\delta, n)<\delta$ is in $N$ for all $n$.) Assume that $\nu \in \bigcap_{n \in \omega} A\left(w_{n}\right)$. Then $\nu \geq \operatorname{seq}(\delta, n)$ for all $n$, so $\nu \geq \delta$. On the other hand, $g_{n}(\nu) \in N$ for all $n$, so $\nu \leq \delta$. But $\delta \notin A\left(w_{0}\right)$, a contradiction.

Of course this shows the following: $\mathfrak{b}\left(\mathrm{PD}^{\emptyset}(I)\right)$ implies $\mathfrak{b}\left(\mathrm{PD}^{\emptyset}\left(I \uparrow E_{>\aleph_{0}}^{\kappa}\right)\right.$ ) (since empty can just cut $\kappa$ into $E_{\aleph_{0}}^{\kappa}$ and $E_{>\aleph_{0}}^{\kappa}$ as a first move).

Recall that $\mathfrak{b}\left(\mathrm{PD}^{\emptyset}(I)\right)$ for any $I$ implies $\mathfrak{b}\left(\mathrm{PD}^{\emptyset}(\mathrm{NS})\right)$ (due to monotonicity). So the last lemma gives: 
Corollary 5.4. $\mathfrak{b}\left(P D^{\emptyset}(I)\right)$ is equivalent to $\mathfrak{b}\left(P D^{\emptyset}\left(I \uparrow E_{>\aleph_{0}}^{\kappa}\right)\right)$ and implies $\mathfrak{b}\left(P D^{\emptyset}(\mathrm{NS})\right)$ and $\mathfrak{b}\left(P D^{\emptyset}\left(\mathrm{NS}\left\lceil E_{>\aleph_{0}}^{\kappa}\right)\right)\right.$.

Lemma 5.5. $\mathfrak{b}\left(P D^{\emptyset}(I)\right)$ implies that I is normally $\infty$-semi precipitous. $\mathfrak{b}\left(c \mathcal{E} c^{\text {min }}(I,<\kappa)\right)$ implies that I is $\infty$-semi precipitous.

Proof. We define the $P^{*}$-name $\underset{\sim}{U}$ by $X \in \underset{\sim}{U}$ iff $X \supseteq A(w)$ for some $w \in G_{P^{*}}$.

- $P^{*}$ forces that $\underset{\sim}{U}$ is a $V$-ultrafilter: Given any $w \in P^{*}$ and $X \in V$, player empty can respond to $w$ by cutting into $X$ and $A(w) \backslash X$.

- In the $c \& \mathrm{c}^{\mathrm{min}}$ case, $P^{*}$ forces that $\underset{\sim}{U}$ is $<\kappa$-complete: Assume that (in $V$ ) $X$ is the disjoint union of $\left(X_{i}\right)_{i \in \lambda}, \lambda<\kappa$. Then empty can responds to $w$ by cutting into $\left\{X_{i}: i \in \lambda\right\} \cup\{A(w) \backslash X\}$.

- In the case of $\mathrm{PD}, P^{*}$ forces that $\underset{\sim}{U}$ is $V$-normal: If $f \in V$ is regressive, then empty can play $f$ as response to any $w$.

- $P^{*}$ forces that $\underset{\sim}{U}$ is wellfounded: Assume towards a contradiction that $w$ forces that $\left(f_{n}\right)_{n \in \omega}$ are functions (in $V$ ) from $\kappa$ to the ordinals such that

$$
\underset{\sim}{A}=\left\{\alpha: \underset{\sim}{f_{n+1}}(\alpha)<\underset{\sim}{f_{n}}(\alpha)\right\}
$$

is in $\underset{\sim}{U}$ for all $n \in \omega$. Set $w_{-1}=w$. Assume that we already have $w_{n}$ (for $n \geq-1$ ). Pick some $w_{n+1}^{\prime}<w_{n}$ deciding $f_{n+1}$ to be some $f_{n+1}^{\prime} \in$ $V$. So $w_{n+1}^{\prime}$ forces that $X_{n+1}:=\bigcap_{l \leq n+1} A_{\sim}=\bigcap_{l \leq n+1} A_{l}^{\prime}$ (a set in $V$ ) is in $U$. In particular, there is some $w_{n+1}$ stronger than $w_{n+1}^{\prime}$ such that $A\left(w_{n+1}\right) \subseteq X_{n+1}$. The sequence $\left(w_{n}\right)_{n \in \omega}$ corresponds to a run of the game. Since nonempty follows the strategy, there is some $\alpha \in \bigcap_{n \in \omega} A\left(w_{n}\right) . w_{n+1}$ forces $\alpha \in X_{n+1}$, i.e., $f_{n+1}^{\prime}(\alpha)<f_{n}^{\prime}(\alpha)$. This gives an infinite decreasing sequence, a contradiction.

Together with 4.4, we get:

Corollary 5.6. In $L[U]$, nonempty does not win $P D^{\emptyset}\left(\mathrm{NS}_{\kappa} \uparrow S\right)$ for any $S \notin U$. In particular, $\mathfrak{b}\left(P D\left(\mathrm{NS}_{\kappa}\right)\right)$ holds (even for the game of length $\left.\kappa\right)$, but $\mathfrak{b}\left(P D_{e}^{\emptyset}\left(\mathrm{NS}_{\kappa}\lceil S)\right)\right.$ fails for every stationary $S$. Also, and $\mathfrak{a}\left(I d\left(\mathrm{NS}_{\kappa}\lceil S)\right)\right.$ fails, i.e., $\mathrm{NS}_{\kappa}$ is nowhere precipitous.

We can use a Levy Collapse to reflect this situation down to, e.g., $\aleph_{2}$. We first list some properties of the Levy collapse. Assume that $\kappa$ is inaccessible, $\theta<\kappa$ regular, and let $Q=\operatorname{Levy}(\theta,<\kappa)$ be the Levy collapse of $\kappa$ to $\theta^{+}:$A condition $q \in Q$ is a function defined on a subset of $\kappa \times \theta$, such that $|\operatorname{dom}(q)|<\theta$ and $q(\alpha, \xi)<\alpha$ for $\alpha>1,(\alpha, \xi) \in \operatorname{dom}(q)$ and $q(\alpha, \xi)=0$ for $\alpha \in\{0,1\}$. Given $\alpha<\kappa$, define $Q_{\alpha}=\{q: \operatorname{dom}(q) \subseteq \alpha \times \theta\}$ and $\pi_{\alpha}: Q \rightarrow Q_{\alpha}$ by $q \mapsto q \uparrow(\alpha \times \theta)$. The following is well known:

- If $q \Vdash p \in G$, then $q \leq p$ (i.e. $\leq *$ is the same as $\leq$ ).

- $Q$ is $\kappa$-cc and $<\theta$-closed.

- In particular, if $p$ forces that $C \subseteq \kappa$ is club, then there is a club $C_{0} \in V$ such that $p$ forces $C_{0} \subseteq C$. The ideal generated by $\mathrm{NS}_{\kappa}^{V}$ in $V[G]$ is $N S_{\kappa}^{V[G]}$.

We also need the following simple fact (see, e.g., [15, 6.2] for a proof):

Let $I$ be a normal ideal concentrated on $E_{\geq \theta}^{\kappa}$, let $T$ be $I$-positive, $p \in Q$ and $p_{\alpha} \leq p$ for all $\alpha \in T$. Then there is an $I$-positive $T^{\prime} \subseteq T$ and a $q \leq p$ such that $\pi_{\alpha}\left(p_{\alpha}\right)=q$ for all $\alpha \in T^{\prime}$. 
So in particular, every $q^{\prime} \leq q$ is compatible with $p_{\alpha}$ for all but boundedly many $\alpha \in T^{\prime}$.

We will also use:

Lemma 5.7. Let $\kappa$ be inaccessible and $T \subset \kappa$ be stationary. The Levy collapse preserves $\neg \mathfrak{b}\left(P D^{\emptyset}\left(\mathrm{NS}_{\kappa}\lceil T)\right)\right.$. The same holds for $P D$.

Proof. Assume towards a contradiction that $q$ forces that nonempty does have a winning strategy in $V[G]$. We describe a winning strategy in $V$ : Assume empty plays $f_{0}$ (in $[V]$ ). Let $q_{0} \leq q$ decide that in $V[G]$ nonempty chooses $\alpha_{0}$ as response to $f_{0}$ according to the winning strategy in $V[G]$. So $q_{0}$ forces that $f_{0}^{-1}\left(\alpha_{0}\right) \cap T$ is stationary, therefore $f_{0}^{-1}\left(\alpha_{0}\right) \cap T$ is stationary in $V$. Generally, let $q_{n} \leq q_{n-1}$ decide that nonempty plays $\alpha_{n}$ as response to $f_{n}$. Since $Q$ is $\sigma$-closed, there is a $q_{\omega}<q_{n}$ for all $n$. So $q_{\omega}$ forces that $\bigcap f_{n}^{-1}\left(\alpha_{n}\right) \cap T$ is stationary.

Starting with $L[U]$ and using a Levy collapse we get:

Corollary 5.8. Consistently relative to a measurable, $\mathfrak{b}\left(P D\left(\mathrm{NS}_{\aleph_{2}}\right)\right.$ ) holds (even for length $\left.\aleph_{1}\right)$ but $\mathfrak{b}\left(P D_{e}^{\emptyset}\left(\mathrm{NS}_{\aleph_{2}} \mid S\right)\right)$ fails for every stationary $S$, and $\mathrm{NS}_{\aleph_{2}}$ is nowhere precipitous.

Proof. Assume $V=L[U]$ and let $Q=\operatorname{Levy}\left(\aleph_{1},<\kappa\right)$ be the Levy collapse of $\kappa$ to $\aleph_{2}$.

To see that $\mathrm{NS}_{\aleph_{2}}$ is forced to be nowhere precipitous, note that $<\kappa$-cc implies $\mathrm{cl}^{V[G]}\left(\mathrm{NS}_{\kappa}^{V}\right)=\mathrm{NS}_{\kappa}^{V[G]}$ and use 4.5 .

In $V[G], \mathrm{cl}^{V[G]}(U)$ is a normal filter such that the family of positive sets has a $\sigma$-closed dense subset [5]. Let $I$ be the dual ideal. So nonempty wins $\operatorname{BM}(I)$, and therefore $\mathrm{PD}_{\mathrm{e}}(I)$ and $\mathrm{PD}\left(\mathrm{NS}_{\kappa}\right)$ (even of length $\left.\aleph_{1}\right)$.

It remains to be shown that $\mathfrak{b}\left(\mathrm{PD}_{\mathrm{e}}^{\emptyset}\left(\mathrm{NS}_{\aleph_{2}} \uparrow S\right)\right)$ fails in $V[G]$ for all stationary $S$. Assume towards a contradiction that some $p$ forces that $S$ is stationary and $\mathfrak{b}\left(\mathrm{PD}^{\emptyset}\left(\mathrm{NS}_{\kappa}\left\lceil S^{\prime}\right)\right)\right.$ holds for all stationary $S^{\prime} \subseteq S$. According to $[5.4$ we can assume $S \subseteq E_{\aleph_{1}}^{\aleph_{2}}$. Set

$$
T_{0}=\{\alpha \in \kappa: p \not \forall \propto \notin \underset{\sim}{S}\}
$$

$T_{0} \subseteq E_{\geq \aleph_{1}}^{\kappa}$ is stationary. Fix some stationary $T \subseteq T_{0}$ not in $U$; and for $\alpha \in T$ fix some $p_{\alpha} \leq p$ forcing $\alpha \in \underset{\sim}{S}$. Apply (6) to $T$, the nonstationary ideal and $\left(p_{\alpha}\right)_{\alpha \in T}$. This results in $q \leq p$ and $T^{\prime} \subseteq T$ stationary.

$$
q \Vdash S^{\prime}:=T^{\prime} \cap \underset{\sim}{S} \text { is stationary. }
$$

Otherwise some $q_{1} \leq q$ forces that $S^{\prime}$ is nonstationary. Then there is in $V$ a club $C$ and a $q_{2} \leq q_{1}$ forcing that $S^{\prime} \cap C=\emptyset$. Pick $\alpha \in T^{\prime} \cap C$ such that $p_{\alpha}$ and $q_{2}$ are compatible. Then $q_{3} \leq p_{\alpha}, q_{2}$ forces that $\alpha \in T^{\prime} \cap C \cap S$, a contradiction. This shows (7).

By our assumption, $p$ forces that nonempty wins $\mathrm{PD}^{\emptyset}\left(\mathrm{NS} \uparrow S^{\prime}\right)$. But $\mathfrak{b}\left(\mathrm{PD}^{\emptyset}\left(\mathrm{NS}_{\kappa} \uparrow\right.\right.$ $\left.T^{\prime}\right)$ ) fails in $V$ (since $T^{\prime} \subset T$ and $\left.T \notin U\right)$, therefore $\mathfrak{b}\left(\mathrm{PD}^{\emptyset}\left(\mathrm{NS}_{\aleph_{2}}\left\lceil T^{\prime}\right)\right.\right.$ ) fails in $V[G]$ according to 5.7, and by monotonicity $\mathfrak{b}\left(\mathrm{PD}^{\emptyset}\left(\mathrm{NS}_{\aleph_{2}}\left\lceil S^{\prime}\right)\right)\right.$ fails as well, a contradiction.

We will now force nonempty not to win PD. For simplicity we will assume CH and look at $\kappa=\aleph_{2}$. It turns out that it is enough to add $\aleph_{1}$ many Cohen reals (actually, many similar forcings also work). First we need another variant of (4) or (5): 
Lemma 5.9. Assume $C H$ and $\mathfrak{b}\left(P D^{\emptyset}\left(\mathrm{NS}_{\aleph_{2}}\right)\right)$. For each $v \in P^{*}$ there are $F^{\prime}(v) \leq v$ and $F^{\prime \prime}(v) \leq v$ such that $A\left(F^{\prime}(v)\right)$ and $A\left(F^{\prime \prime}(v)\right)$ are disjoint.

(We can choose $F^{\prime}(v)$ and $F^{\prime \prime}(v)$ to be immediate successors of $v$, i.e. we just have to choose two regressive functions $f^{\prime}$ and $f^{\prime \prime}$ as empty's moves.)

Proof. We fix an injection $\phi:\left[\aleph_{2}\right]^{\aleph_{0}} \rightarrow \aleph_{2}$. Let $S=C \cap E_{\omega_{1}}^{\aleph_{2}}$ (for some clubset $C$ ) consist of ordinals $\alpha$ such that $\phi^{\prime \prime}[\alpha]^{\aleph_{0}} \subseteq \alpha$. For each $\alpha \in S$, pick a normal cofinal sequence $\gamma^{\alpha}: \omega_{1} \rightarrow \alpha$. For $i \in \omega_{1}$ set $g_{i}(\alpha)=\phi\left(\left\{\gamma^{\alpha}(j): j \leq i\right\}\right)$ for $\alpha \in S$; and set $g_{i}(\alpha)=0$ for $\alpha \notin S$. So for all $i \in \omega_{1}, g_{i}$ is a regressive function. If $\alpha \neq \beta$ then $g_{i}(\alpha) \neq g_{i}(\beta)$ for some $i$; and $g_{i}(\alpha) \neq g_{i}(\beta)$ implies $g_{j}(\alpha) \neq g_{j}(\beta)$ for all $j>i$.

Let $x(i)$ be the strategy's response to $v^{\frown} g_{i}$, We can identify $x(i)$ with the sequence $\phi^{-1} x(i)=\left(\gamma_{i, k}\right)_{k \leq i}$. So for all $\alpha$ with $g_{i}(\alpha)=x(i)$ we get $\gamma^{\alpha}(k)=\gamma_{i, k}$ for $k \leq i$.

Case A: There are $k<i<j<\omega_{1}$ such that $\gamma_{i, k} \neq \gamma_{j, k}$. Then set $F^{\prime}=g_{i}$ and $F^{\prime \prime}=g_{j}$. If $\alpha \in g_{i}^{-1}(x(i))$ and $\beta \in g_{j}^{-1}(x(j))$, then $\gamma^{\alpha}(k) \neq \gamma^{\beta}(k)$, so in particular $\alpha \neq \beta$.

Case B: Otherwise, all the sequences $\left(\gamma_{i, k}\right)_{i \leq k}$ cohere for all $i \in \omega_{1}$, so let $\left(\tilde{\gamma}_{k}\right)_{k \in \omega_{1}}$ be the union of these sequences, with supremum $\tilde{\alpha}<\omega_{2}$. So for all $\alpha \neq \tilde{\alpha}$ in $S$ there is some $k(\alpha) \in \omega_{1}$ such that $\gamma^{\alpha}(k(\alpha)) \neq \tilde{\gamma}_{k(\alpha))}$. Set $k(\alpha)=0$ for $\alpha \in \omega_{1} \cup\{\tilde{\alpha}\} \cup \omega_{2} \backslash S$. So $k$ is a regressive function. Let $l$ be the strategy's response to $v \frown k$. Set $F^{\prime}=k$ and $F^{\prime \prime}=g_{l}$. If $\alpha \in k^{-1}(l)$ and $\beta \in g_{l}^{-1} x(l)$, then $\gamma^{\beta}(l)=\tilde{\gamma}_{l}$ which is different to $\gamma^{\alpha}(l)$.

Lemma 5.10. Assume CH. Let $P_{\omega_{1}}$ be the forcing notion adding $\aleph_{1}$ many Cohen reals. Then $P_{\omega_{1}}$ forces $\neg \mathfrak{b}\left(P D^{\emptyset}\left(\mathrm{NS}_{\aleph_{2}}\right)\right)$.

(The same holds for any other $\mathrm{CH}$ preserving $\omega_{1}$-iteration of absolute ccc forcing notions.) Note that since $\mathrm{PD}^{\emptyset}$ is monotone, $\mathfrak{b}\left(\mathrm{PD}^{\emptyset}(I)\right)$ fails for all ideals $I$ on $\aleph_{2}$.

Proof. Assume that $p \in P_{\omega_{1}}$ forces that $\tau$ is a winning strategy for nonempty for the game $\operatorname{PD}^{\emptyset}(I)$.

Let $P_{\alpha}$ be the complete subforcing of the first $\alpha$ Cohen reals. $P_{\omega_{1}}$ forces that Lemma 5.9 holds. We fix the according $P_{\omega_{1}}$-names $\underset{\sim}{F^{\prime}}$ and ${\underset{\sim}{F}}^{\prime \prime}$. Let $N \prec H(\chi)$ be countable and contain $p, \tau, \underset{\sim}{F}$ and ${\underset{\sim}{*}}^{\prime \prime}$. Set $\epsilon=N \cap \omega_{1}$. If $\tilde{G}_{\omega_{1}}$ is $P_{\omega_{1}}$-generic over $V$, then $G_{\epsilon}=G_{\omega_{1}} \cap P_{\epsilon}$ is $P_{\omega_{1}}$-generic over $N$ (and $P_{\epsilon}$-generic over $V$ ).

So in $N_{\epsilon}=N\left[G_{\epsilon}\right]=N\left[G_{\omega_{1}}\right]$, we can evaluate the correct values of $\tau, \underset{\sim}{F^{\prime}}$ and ${\underset{\sim}{F}}^{\prime \prime}$ for all valid sequences $v$ in $N_{\epsilon}$ (i.e., the resulting values are the same as the ones calculated in $\left.V_{\omega_{1}}=V\left[G_{\omega_{1}}\right]\right)$.

In $V_{\omega_{1}}$, pick any real $r \notin V_{\epsilon}$. Using $r$, we now define by induction a run $b$ of the game such that each initial segment is in $N_{\epsilon}$ : Assume we already have the valid sequence $u \in N_{\epsilon}$. Extend $u$ with $\underset{\sim}{F^{\prime}}(u)$ if $r(n)=0$, and to $F^{\prime \prime}(u)$ otherwise.

So $b \in V_{\omega_{1}}$ is a run of the game according to $\tau$; nonempty wins the run; so there is some $\delta \in \bigcap_{n \in \omega} A\left(b\lceil n)\right.$. But we can in $V_{\epsilon}$ use this $\delta$ to reconstruct (by induction) the run $b$ and therefore the real $r$ : Assume we already know $r\lceil n$ and the corresponding valid sequence $u=b\lceil n$. Then $\delta$ is element of exactly one of $A\left(F^{\prime}(u)\right)$ or $A\left(F^{\prime \prime}(u)\right)$, which determines $r(n)$ as well as the sequence corresponding to $b\lceil(n+1)$.

On the other hand, adding Cohens, as any $\kappa$-cc forcing, preserves precipitousness (and non-precipitousness) of an ideal, cf. 4.5, So we get: 
Corollary 5.11. $\mathfrak{a}(I d(I))$ does not imply $\mathfrak{b}\left(P D^{\emptyset}(\mathrm{NS})\right)$.

If we assume $\mathrm{CH}$ and an $\aleph_{3}$-saturated normal ideal on $\aleph_{2}$ saturated on $E_{\aleph_{1}}^{\aleph_{2}}$, we get the following:

Corollary 5.12. (Saturated ideal.) $\mathfrak{a}(B M(I))$ does not imply $\mathfrak{b}\left(P D^{\emptyset}(\mathrm{NS})\right)$.

Proof. Since $P_{\omega_{1}}$ has size $\aleph_{1}<\aleph_{2}, \operatorname{cl}(I)$ remains $\aleph_{3}$-saturated. So in $V[G]$, we can use 3.4 to see that $\mathfrak{a}(\mathrm{BM}(\mathrm{cl}(I)))$ holds.

\section{6. $\mathfrak{b}\left(\mathrm{PD}_{\mathrm{e}}\right)$ FOR A NONPRECIPITOUS $I$.}

We have seen that $\mathfrak{b}(\mathrm{PD}(I))$ can hold for a nowhere precipitous ideal $I$. It is a bit harder to show that there can be a nowhere precipitous ideal $I$ that even satisfies $\mathfrak{b}\left(\mathrm{PD}_{\mathrm{e}}(I)\right)$.

Fact 6.1. The following is consistent relative to $\kappa$ measurable: $I_{0}$ is nowhere precipitous, and for every $I_{0}$-positive set $S$ the dual to $I_{0} \uparrow S$ can be extended to a normal ultrafilter.

Note that this implies $\mathfrak{b}\left(\mathrm{PD}_{\mathrm{e}}\left(I_{0}\right)\right)$, even for the game of length $\kappa$.

And as usual, we can use a Levy collapse to reflect these properties to $\aleph_{2}$ :

Lemma 6.2. Start with a universe $V$ as in Fact 6.1. After collapsing $\kappa$ to $\aleph_{2}$, we get: $\operatorname{cl}(I)$ is nowhere precipitous and satisfies $\mathfrak{b}\left(P D_{e}(\mathrm{cl}(I))\right)$ (even for the game of length $\left.\aleph_{1}\right)$.

Proof. Nowhere precipitous follows from 4.5. Let $S$ be a $P$-name for a $\operatorname{cl}(I)$-positive set and $p \in P$. Will show:

In $V$ there is a normal ultrafilter $U$ and a $q \leq p$ forcing that $S$ is $\operatorname{cl}(U)$-positive.

Then according to the usual argument, the $\operatorname{cl}(U)$-positive sets have a $\sigma$-closed dense subset, so nonempty wins $\operatorname{BM}(\operatorname{cl}(U) \uparrow S)$, and - since $\operatorname{cl}(U)$ extends $\operatorname{cl}(I)$ - nonempty wins $\operatorname{PD}\left(I\lceil S)\right.$ (even for length $\aleph_{1}$ ).

To prove (8), set $T=\left\{\alpha \in E_{>\aleph_{1}}^{\kappa}: p \| \forall \notin S\right\}$. $T$ is $I$-positive. For each $\alpha \in T$ pick a witness $p_{\alpha} \leq p$. Let $q, T^{\prime \prime}$ be as in (6) and pick a normal ultrafilter $U$ containing $T^{\prime \prime}$. We have to show that $q$ forces $S$ to be $\operatorname{cl}(U)$-positive. Assume otherwise, and pick $q^{\prime} \leq q$ and $A \in U$ such that $q^{\prime}$ forces $A \cap S=\emptyset$. Then $q^{\prime} \in Q_{\alpha}$ for some $\alpha<\kappa$. Pick $\beta \in T^{\prime \prime} \cap A \backslash \alpha$. Then $p_{\beta}$ and $q^{\prime}$ are compatible, a contradiction to $p_{\beta} \Vdash \beta \in S$.

After this paper was submitted, it came to our attention that Fact 6.1 follows directly from a construction of Gitik, using only a measurable: In his paper Some pathological examples of precipitous ideals [6], he constructs a non-precipitous filter $U^{*}$ as intersection of normal ultrafilters (see page 502 and Lemma 3.3).

We still give our proof of Fact 6.1 in the rest of the paper, using a supercompact and assuming GCH in the ground model, since the construction itself might be of some interest.

We will split the proof into several lemmas: First we define the forcing $S(\kappa)$ as limit of $P_{\alpha}$. We also define dense subsets $P_{\alpha}^{\prime}$ of the $P_{\alpha}$. Then we define the forcing notion $R_{\kappa+1}$, by doing the usual Silver-style preparation with reverse Easton support. This forcing notion is as required: In the extension, we define 6.6 the ideal $I_{0}$ and show that Fact 6.1 holds 6.9 and 6.10). 
6.1. The basic forcing. So let us assume that $\kappa$ is an inaccessible cardinal, and define $S(\kappa)$ as the limit of the $<\kappa$-support iteration $\left(P_{a}, Q_{a}\right)_{a \in \kappa^{+}}$of length $\kappa^{+}$ defined the following way: By induction on $a$, we define $Q_{a}$ together with the $P_{a}$-names $B_{a} \subseteq \kappa, g_{a}: \kappa \rightarrow \kappa+1$ and the $P_{a+1}$-names $A_{a} \subseteq \kappa, f_{a}: \kappa \rightarrow \kappa$ :

We identify the tree $T=\left(\kappa^{+}\right)^{<\omega}$ of finite $\kappa^{+}$-sequences with $\kappa^{+}$such that the root is identified with 0 . We can assume that $a<_{T} b$ implies $a<b$ (as ordinals in $\kappa^{+}$). We write $a \triangleleft_{T} b$ or $b \triangleright_{T} a$ to denote that $b$ is immediate $T$-successor of $a$. So for all $a \in \kappa^{+}$there are $\kappa^{+}$many $b$ with $a \triangleleft_{T} b$. For $b \neq 0$ we also write $\operatorname{prec}(b)$ to denote the (unique) $a$ such that $a \triangleleft_{T} b$.

Assume we already have defined $P_{a}$, and the $P_{b+1}$-names $A_{b}, f_{b}$ for all $b<a$. Then in $V\left[G_{P_{a}}\right]$, we define $B_{a}, g_{a}, Q_{a}$ and the $Q_{a}$-names $f_{a}, A_{a}$ :

- If $a=0$, we set $g_{a}(\alpha)=\kappa$ for all $\alpha \in \kappa$, and $B_{a}=\kappa$.

- Otherwise, we use some bookkeepingl to find a $B_{a}^{0} \subseteq A_{\operatorname{prec}(a)}$, and we set:

$$
B_{a}=B_{a}^{0} \backslash \nabla_{b<a: \operatorname{prec}(b)=\operatorname{prec}(a)} A_{b} \text {, and we set } g_{a}=f_{\operatorname{prec}(a)} .
$$

- A condition $p$ of $Q_{a}$ is a function $f^{p}: \beta^{p} \rightarrow \kappa$ such that $\beta^{p} \in \kappa$ and for all $\alpha \in \beta^{p}$ :

- if $\alpha \notin B_{a}$ or $g_{a}(\alpha)=0$ then $f^{p}(\alpha)=0$,

- otherwise $f^{p}(\alpha)<g_{a}(\alpha)$.

- Additionally, if $a=0$ we require $f^{p}(\alpha)>0$.

- We define the order on $Q_{a}$ by $q \leq p$ if $f^{q} \supseteq f^{p}$.

- We set $f_{a}$ to be the canonical $Q_{a}$-generic, i.e., $\bigcup_{q \in G} f^{q}$.

- We set $A_{a}=\{\alpha \in \kappa: f(\alpha)>0\}$. (So $A_{0}=\kappa$, and $A_{a} \subseteq B_{a} \subseteq B_{a}^{0}$.)

Note that to write the diagonal union in (9), we have to identify the index set with $\kappa$. Different identifications lead to the same result modulo club. In particular, we get:

$$
\text { If } b<a \text { and } \operatorname{prec}(b)=\operatorname{prec}(a) \text { then } B_{a} \cap A_{b} \text { is nonstationary. }
$$

Obviously $Q_{a}$ is $<\kappa$-closed. We now define $P_{a}^{\prime}$ by induction on $a \in \kappa^{+}$and show (in the same induction) that $P_{a}^{\prime}$ is $<\kappa$-closed and can be interpreted to be a dense subset of $P_{a}$. A condition $p \in P_{a}^{\prime}$ is a function from $u \times \beta$ to $\kappa$ such that:

- $\beta \in \kappa$.

- $u$ is a subset of $a$ of size $<\kappa$.

- $c \triangleleft_{T} b$ implies $\max (1, p(c, \alpha))>p(b, \alpha)$.

- $p(b, \alpha)>0$ implies that $p\left\lceil b\right.$ forces (as element of $\left.P_{b}\right) \sqrt{9}$ that $\alpha \in B_{b}$.

- If $0 \in u$, then $p(0, \alpha)>0$ for all $\alpha<\beta$.

We can interpret $p \in P_{a}^{\prime}$ to be a condition in $P_{a}$ in the obvious way; in particular we can define the order on $P_{a}^{\prime}$ to be the one inherited from $P_{a}$.

Lemma 6.3. $\quad P_{a}^{\prime}$ is a dense subset of $P_{a}$.

- The order on $P_{a}^{\prime}$ (as inherited from $P_{a}$ ) is the extension relation.

- $P_{a}^{\prime}$ is $<\kappa$-closed.

- $P_{a}$ is strategically $<\kappa$-closed.

Proof. By induction on $a$ (formally, the definition of $P_{a}^{\prime}$ has to be done in the same induction as well). It is clear that $P_{a}^{\prime}$ is closed and that the order is extension. We

\footnotetext{
${ }^{8}$ We just need to guarantee that $P_{\kappa}+$ forces: For every $a \in T$ and every subset $B$ of $A_{a}$ there is a $b \triangleright_{T} a$ such that $B_{b}^{0}=B$. Note that $A_{b} \subseteq B \subseteq A_{a}$.

${ }^{9}$ by induction, we already know that $P_{b}^{\prime}$ is dense in $P_{b}$
} 
have to show that $P_{a}^{\prime}$ is dense in $P_{a}$. We do that by case distinction on $\operatorname{cf}(a)$ :

The case $\operatorname{cf}(a) \geq \kappa$ is trivial.

The successor case: Assume $a=b+1$ and $p \in P_{a}$. Then by induction we know that $P_{b}$ is strategically $\kappa$ closed, so we can strengthen $p\left\lceil b\right.$ to some $p^{\prime} \in P_{b}^{\prime}$ deciding $p(b)$ to be some $f^{p}$. We can assume that the height of $p^{\prime}$ is at least the height of $f^{p}$, and we can extend $f^{p}$ up to the height of $p^{\prime}$ by adding zeros on top. Then $p^{\prime}$ together with $f^{p}$ is a condition of $P_{a}^{\prime}$ stronger than $p$.

The case $\operatorname{cf}(a)<\kappa$, i.e., $a=\sup \left(b_{i}: i \in \lambda\right)$ for some $b_{i}<a$ and $\lambda<\kappa$. We assume $p \in P_{a}$. We define by induction on $i \in \lambda$ decreasing conditions $p_{i}^{\prime} \in P_{b_{i}}^{\prime}$ stronger than $p\left\lceil b_{i}\right.$. (By induction we know that $P_{b_{i}}$ is $<\kappa$-closed, so $\tilde{p}_{i}=\bigcup_{l<i} p_{l}^{\prime}$ is in $P_{b_{i}}$ and, by induction, stronger than $p\left\lceil\sup _{l<i} b_{l}\right.$. So we can extend $\tilde{p}_{i}$ to an element of $P_{b_{i}}$ stronger than $p\left\lceil b_{i}\right.$.)

6.2. The Silver style iteration. We now use the basic forcing $S(\kappa)$ in a reverse Easton iteration, the first part acting as preparation to allow the preservation of measurability. This method was developed by Silver to violate GCH at a measurable, and has since been established as one of the basic tools in forcing with large cardinals. We do not repeat all the details here, a more detailed account can be found in [10, 21.4]. Note that here we do not just need to preserve measurability or supercompactness (for this, we could just use Laver's general result [16]), we need specific properties of the Silver iteration.

Fix a $j: V \rightarrow M$ such that

$$
M \text { is closed under } \kappa^{++} \text {-sequences. }
$$

In particular, $\operatorname{cf}(j(\kappa))>\kappa^{+}$.

We will use the reverse Easton iteration $\left(R_{a}, S(a)\right)_{a \leq \kappa}$, for $S(a)$ defined as above. $R_{\kappa}$ is the preparation that allows us to preserve measurability (and we will not need it for anything else); we will look at $R_{\kappa} * P_{a}$ for $a \leq \kappa^{+}$, and in particular at $R_{\kappa+1}=R_{\kappa} * P_{\kappa^{+}}$(recall that $S(\kappa)=P_{\kappa^{+}}$). We claim that $R_{\kappa+1}$ forces what we want. We will also use $j\left(R_{\kappa} * P_{a}\right) \in M$. We get the usual properties:

- The definition of $R$ is sufficiently absolute. In particular, we can (in $M$ ) factorize $j\left(R_{\kappa+1}\right)=R_{j(\kappa)+1}$ as $R_{\kappa+1} * R^{\prime}$, where $R^{\prime}$ is the quotient forcing $R_{j(\kappa)+1}^{\kappa+1}$. Note that $R^{\prime}$ is $<\kappa^{+++}$-closed (in $M$ and therefore in $V$ as well).

- Assume that $G$ is $R_{\kappa+1}$-generic over $V$ (and $M$ ). $M[G]$ is closed (as subset of $V[G]$ ) under $\kappa^{+}$-sequences. In particular, $\kappa^{+}$is the same (and also equal to $2^{\kappa}$ ) in $V, V[G]$ and $M[G]$.

- For $p \in R_{\kappa+1}$, the domain of $j(p)$ is in $\kappa \cup\{j(\kappa)\}$, moreover $j(p)\lceil\kappa=p\lceil\kappa$ and $j(p)(j(\kappa))$ is isomorphic to $p(\kappa)$ such that $a \in \operatorname{dom}(p(\kappa))$ is mapped to $j(a)$. The image of $G$ under $j$ is element of $V[G]$ and subset of $M$ of size $\kappa^{+}$, therefore element of $M[G]$. For $p \in G$ we can split in $M$ the condition $j(p)$ into $p\lceil\kappa$ (which is in $G$ anyway) and $j(p(\kappa))$. We can assume that $G$ actually is $R_{\kappa} * P_{\kappa^{+}}^{\prime}$-generic (since $P_{\kappa^{+}}^{\prime}$ is dense in $P_{\kappa^{+}}$). Then $j(p(\kappa))$ is a $P_{j\left(\kappa^{+}\right)^{-}}^{\prime}$-condition. So in $M[G]$, the set $\{j(p(\kappa)): p \in G\}$ is a directed subset of $P_{j\left(\kappa^{+}\right)}^{\prime}$ of size $\kappa^{+}$, therefore the union is a $P_{j\left(\kappa^{+}\right)^{-}}^{\prime}$-condition $q_{G}$, a matrix of height $\kappa$ (which is less than $j(\kappa)$, so no contradiction to the definition of $P_{a}^{\prime}$ ) and with domain $j^{\prime \prime} \kappa^{+}$(which has size $\kappa^{+}<j(\kappa)^{M[G]}$ ). We call this condition $q_{G}$, the minimal $G$-master condition.

- In $M[G]$, we call $q \in R^{\prime}$ a $G$-master condition if it is stronger than $q_{G}$. 
- If $H$ contains some $G$-master condition and is $R^{\prime}$-generic over $V[G]$ (and therefore $M[G]$ as well), then we can extend in $V[G][H]$ the embedding $j$ to $V[G] \rightarrow M[G][H]$ by setting $j(\tau[G])=j(\tau)[G][H]$. This defines in $V[G][H]$ a normal ultrafilter $U=\{A[G]: \kappa \in j(A)[G][H]\}$ over $V[G]$. Since $R^{\prime}$ is sufficiently closed, $U$ is already element of $V[G]$.

Definition 6.4. In $V[G], a \in \kappa^{+}$is called a positive index, if

$$
(\forall \zeta<j(\kappa))(\exists q G \text {-master condition }) q \Vdash\left(\kappa \in j\left(B_{a}\right) \& j\left(g_{a}\right)(\kappa)>\zeta\right) .
$$

Otherwise, $a$ is called a null-index.

Here we interpret $B_{a}$ and $g_{a}$ as $R_{\kappa} * P_{a}$-names in the canonical way, so the $j$ images are $R_{j(\kappa)} * P_{j(a)}$-names. In particular, whether $\kappa \in j\left(B_{a}\right) \& j\left(g_{a}\right)(\kappa)>\zeta$ holds is already decided in the $R_{j(\kappa)} * P_{j(a)}$ extension, so we can assume that the $G$-master condition $q$ of the definition only consists of the required minimal master condition $q_{G}$ "from $j(a)$ onwards", more exactly we can assume:

- $q \in R_{j(\kappa)+1}$ is factorized as $x * y$, for $x \in R_{j(\kappa)}$ and $y$ is $R_{j(\kappa)}$-name for a condition in $P_{j\left(\kappa^{+}\right)}^{\prime}$.

- $x$ forces that $(j(b), \alpha)$ is not in the domain of the matrix $y$ for any $b \geq a$ and $\alpha \geq \kappa$.

In particular, we can extend $q$ to a master condition $q^{\prime}$ forcing that

$$
j\left(f_{b}\right)(\kappa)=0 \text { for all } b \geq a .
$$

Similarly, we can extend $q$ to a master condition $q^{\prime}$ forcing that

$$
j\left(f_{a}\right)(\kappa)=\zeta \text { and } j\left(f_{b}\right)(\kappa)=0 \text { for all } b>a .
$$

Lemma 6.5. If $a$ is null and $b>_{T} a$, then $b$ is null as well. Also, 0 is a positive index.

Proof. Pick $\zeta<j(\kappa)$ such that every master condition forces $j\left(g_{a}\right)(\kappa)<\zeta$ or $\kappa \notin$ $j\left(B_{a}\right)$. But the empty condition forces $j\left(g_{b}\right)(\kappa) \leq j\left(g_{a}\right)(\kappa)$ and $j\left(B_{b}\right) \subseteq j\left(B_{a}\right)$.

Definition 6.6. In $V[G]$, we define the ideal $I_{0}$ by $A \in I_{0}$ iff there is an $X \subseteq \kappa^{+}$ of size $\kappa$ consisting of null-indices such that

$$
A \subseteq \nabla_{i \in X} B_{i} \text { modulo a club set } 10
$$

Lemma 6.7. $I_{0}$ is a normal ideal on $\kappa$

Proof. Assume that $A_{i} \cap C_{i} \subseteq \nabla_{l \in X_{i}} B_{l}$ for all $i \in \kappa$. Then $\left(\nabla_{i \in \kappa} A_{i}\right) \cap \Delta_{i \in \kappa} C_{i} \subseteq$ $\nabla_{l \in \cup X_{i}} B_{l}$ modulo a club set.

By elementarity, if $q$ is a $G$-master condition and if $\varphi\left(c, B_{\alpha}[G], g_{\alpha}[G]\right)$ holds in $V[G]$ for some $c \in V$, then for all $H$ containing $q$ we get in $M[G][H]$

$$
\varphi\left(j(c), j\left(B_{\alpha}\right)[G][H], j\left(g_{\alpha}\right)[G][H]\right) .
$$

Lemma 6.8. In $V[G]$ the following holds: If $a$ is a positive index, then $A_{a}$ is $I_{0}$-positive.

\footnotetext{
${ }^{10}$ As mentioned above, $\nabla_{i \in X} B_{i}$ is only defined modulo a club set, since $X$ is not canonically isomorphic to $\kappa$ (it is just a subset of $\kappa^{+}$of size $\kappa$ ). To avoid ambiguity, we just fix from now on for each such $X$ a bijection to $\kappa$ and make $\nabla_{i \in X} B_{i}$ well defined; still we use "subset modulo club set" in the definition on $I_{0}$.
} 
Note that this implies: $a$ is a positive index iff $B_{a}$ is a $I_{0}$-positive set; and $I_{0}$ is nontrivial (since 0 is a positive index).

Proof. Assume otherwise, and fix an appropriate $X$ and a club set $C$, i.e.,

$$
A_{a} \cap C \subseteq \nabla_{i \in X} B_{i} .
$$

Since $X$ consists of null-indices, there is for each $b \in X$ a $\zeta_{b}<j(\kappa)$ such that every master condition forces $\kappa \notin j\left(B_{b}\right)$ or $j\left(g_{b}\right)(\kappa)<\zeta_{b}$. Since $\operatorname{cf}(j(\kappa))>\kappa$, we can find an upper bound $\xi$ for all $\zeta_{b}$. So every master condition forces

$$
\kappa \in j\left(B_{b}\right) \text { implies } j\left(g_{b}\right)(\kappa)<\xi \text { for all } b \in X \text {. }
$$

Since $a$ is a positive index, we can find a master condition $q$ forcing $\kappa \in j\left(B_{a}\right)$ and $j\left(g_{a}\right)(\kappa)>\xi+1$. According to (14) we can extend $q$ to $q^{\prime}$ such that

$$
j\left(f_{a}\right)(\kappa)>\xi \text { and } j\left(f_{c}\right)(\kappa)=0 \text { for all } c>a .
$$

Since $C$ is club, $\kappa$ is forced to be in $j(C)$. So $q^{\prime}$ forces $\kappa \in j\left(A_{a}\right) \cap j(C)$. According to (17), $A_{a} \cap C \subseteq \nabla_{b \in X} B_{b}$ holds in $V[G]$, so $q^{\prime}$ forces $\kappa \in j\left(A_{a}\right) \cap j(C) \subset j\left(\nabla_{b \in X} B_{b}\right)$. Let $Z$ be the sequence $\left(B_{b}\right)_{b \in X}$. Recall that we fixed (in $V[G]$ ) some bijection $i$ of $\kappa$ to $X$, to make $\nabla Z$ well defined. So $j(\nabla Z)$ uses $j(i)$, a bijection from $j(\kappa)$ to $j(X)$; and $\kappa \in j(\nabla Z)$ means: There is an $\alpha<\kappa$ such that $\kappa \in j(Z)_{j(i)(\alpha)}$. Note that $j(Z)_{j(i)(\alpha)}=j\left(B_{i(\alpha)}\right)$ and set $b=i(\alpha) \in X$. So

$$
\kappa \in j\left(B_{b}\right) \text { for some } b \in X \text { (in particular, } b \text { is null-index). }
$$

We further extend $q^{\prime}$ to some $q^{\prime \prime}$ deciding the $b$ of (20). So $q^{\prime \prime}$ forces

$$
\kappa \in j\left(A_{a} \cap B_{b}\right) \text { for the null-index } b .
$$

We will get a contradiction by case distinction on the position of $b$ relative to $a$ in the tree $T$ :

- $b<_{T} a$ : This contradicts the fact that $b$ is a null-index and $a$ not.

- $a \triangleleft_{T} b$ : Then $g_{b}=f_{a}$, and $q^{\prime \prime}$ forces that $\kappa \in j\left(B_{b}\right)$ and $j\left(g_{b}\right)(\kappa) \geq$ $j\left(f_{a}\right)(\kappa)>\xi>\zeta_{b}$, contradicting (18).

- $a \triangleleft_{T} c$ and $c<_{T} b$ : Then $c$ is (as an ordinal) bigger than $a$, and $q^{\prime \prime}$ forces $\kappa \notin j\left(A_{c}\right)$. So $\kappa \notin j\left(B_{b}\right) \subseteq j\left(A_{c}\right)$.

- So $a$ and $b$ have to be incomparable in $T$, and there is some node $c$ where $a$ and $b$ split. Let $a^{\prime}$ and $b^{\prime}$ the according immediate $T$-successors of $c$. So $a^{\prime} \triangleright_{T} c, b^{\prime} \triangleright_{T} c, a^{\prime} \leq_{T} a, b^{\prime} \leq_{T} b$ and $a^{\prime} \neq b^{\prime}$. Let $\underline{m}$ be the minimum of $a^{\prime}, b^{\prime}$ (as ordinals) and $\bar{m}$ the maximum. According to (10) $A_{m} \cap B_{\bar{m}}$ is nonstationary, so $\kappa \notin j\left(A_{m} \cap B_{\bar{m}}\right)$. So (21) implies that $b^{\prime}=b=$ $\underline{m}$. Also $j\left(g_{b}\right)(\kappa)=j\left(f_{c}\right)(\kappa) \geq j\left(f_{a}\right)(\kappa)>\xi$ according to (19) which contradicts (18).

Lemma 6.9. In $V[G]$, empty has a winning strategy for $I d_{n e}\left(I_{0}\right)$.

Proof. Assume that we have a partial run of the game of length $n$, corresponding to the node $a$ in $T$, and empty has played $X_{n}$ as last move, which is a subset of $A_{a}$. Assume that nonempty plays the $I_{0}$-positive set $B^{0} \subseteq A_{a}$. Let $b \triangleright_{T} a$ be such that $X_{n+1}:=A_{b} \cap B^{0}$ is $I_{0}$-positive, and let $X_{n+1}$ be empty's answer (and $b$ be the new $T$-node corresponding to the new partial run). This is a winning strategy since $f_{n}(\alpha)$ decreases along every branch of $T$. It remains to be shown that we can find a $b \triangleright_{T} a$ as above: $B^{0}$ itself is enumerated as $B_{c}^{0}$ by the bookkeeping at some 
stage $c \triangleright_{T} a$. Recall that $B_{c}=B_{c}^{0} \backslash \nabla_{d<c, d \triangleright_{T} a} A_{d}$. If $B_{c}$ is positive, then we can set $b=c$. Otherwise, since $B_{c}^{0}$ is positive, some $B_{c}^{0} \cap A_{d}$ has to be positive for some $d<c, d \triangleright_{T} a$ (since $I_{0}$ is normal); and we can set $b=d$.

It remains to be shown:

Lemma 6.10. In $V[G]$, for every $I_{0}$-positive $X$ there is a normal ultrafilter $D_{1}$ extending the dual of $I_{0}$ and containing $X$.

Proof. It is enough to show: If $Y$ is $I_{0}$-positive, then there is a master condition $q$ forcing

$$
\kappa \in j(Y) \text { and } \kappa \notin j\left(B_{b}\right) \text { for all null-indices } b \text {. }
$$

Let $X$ be the set of indices $a$ such that $Y \cap A_{a}$ is $I_{0}$-positive. Assume $a \in X$. We will use $Y \cap A_{a}$ as $B_{b}^{0}$ for some $b \triangleright_{T} a$. We have to distinguish two cases:

Case 1: There is a positive $c \triangleright_{T} a$ such that $B_{c} \subseteq Y$. In particular, this will be the case if $b$ itself is positive, i.e. if $B_{b}=B_{b}^{0} \backslash \nabla_{c<b, c \triangleright_{T} a} A_{c}$ is $I_{0}$-positive.

Case 2: There is no such $c$. In particular, in this case $b$ is a null-index, so $Y \cap A_{a}$ is covered (modulo $I_{0}$ ) by $\nabla_{c<b, c \triangleright_{T} a} A_{c}$. Then $c \notin X$ for any $c \geq b$ such that $c \triangleright_{T} a$. So at most $\kappa$ many immediate $T$-successors of $a$ are in $X$; and $Y \cap A_{a}$ is covered (modulo $I_{0}$ ) by $\nabla_{c \triangleright_{T} a, c \in X} A_{c}$ as well.

We claim that Case 1 has to occur for some $a$. Otherwise, $X$ is a subtree of $T$ such that every node has at most $\kappa$ many successors, i.e., there are only $\kappa$ many branches through $X$. By induction on $n, Y$ is covered (module $I_{0}$ ) by $\nabla_{c \in X}, T$-height $(c)=n A_{c}$. But for any branch $b$, the set $\bigcap_{n \in \omega} A_{b(n)}$ is empty (witnessed by the decreasing sequence $\left.f_{b(n)}\right)$, a contradiction.

So we can pick a $T$-minimal $b$ such that Case 1 holds. Note that $\left|j^{\prime \prime} \kappa^{+}\right|<$ $\operatorname{cf}(j(\kappa))$. For every null-index $c$ there is a witness $\xi_{c}<j(\kappa)$, so there is a universal bound $\xi$. Since $b$ is a positive index, we can find a master condition $q$ forcing $j\left(g_{b}\right)(\kappa)>\xi$ and $\kappa \in j\left(B_{b}\right)$. Recall that $B_{b} \subseteq Y\left(\bmod I_{0}\right)$, so $q$ forces that $\kappa \in j(Y)$. We now extend $q$ to $q^{\prime}$ so that it forces $\kappa \notin j\left(A_{c}\right)$ for all $c>b$. Then $q^{\prime}$ is as required: $\kappa \notin j\left(B_{c}\right)$ for any null-index $c$, by a similar case distinction as in the proof of Lemma 6.8 .

\section{REFERENCES}

1. Yoshihiro Abe, Weakly normal ideals on $\mathcal{P}_{\kappa} \lambda$ and the singular cardinal hypothesis, Fund. Math. 143 (1993), no. 2, 97-106. MR MR1240627 (95d:03093)

2. Hans-Dieter Donder and Jean-Pierre Levinski, On weakly precipitous filters, Israel J. Math. 67 (1989), no. 2, 225-242. MR MR1026565 (90j:03093)

3. Asaf Ferber and Moti Gitik, On almost precipitous ideals, Arch. Math. Logic 49 (2010), no. 3, 301-328. MR MR2609985

4. Matthew Foreman, Games played on Boolean algebras, J. Symbolic Logic 48 (1983), no. 3, 714-723. MR MR716633 (85h:03064)

5. F. Galvin, T. Jech, and M. Magidor, An ideal game, J. Symbolic Logic 43 (1978), no. 2, 284-292. MR MR0485391 (58 \#5237)

6. Moti Gitik, Some pathological examples of precipitous ideals, J. Symbolic Logic 73 (2008), no. 2, 492-511. MR MR2414461 (2009a:03052)

7. _ On normal precipitous ideals, Israel J. Math. 175 (2010), 191-219. MR MR2607544

8. Martin Goldstern, A taste of proper forcing, Set theory (Curaçao, 1995; Barcelona, 1996), Kluwer Acad. Publ., Dordrecht, 1998, pp. 71-82. MR MR1601976 (99f:03069)

9. T. Jech, M. Magidor, W. Mitchell, and K. Prikry, Precipitous ideals, J. Symbolic Logic 45 (1980), no. 1, 1-8. MR MR560220 (81h:03097) 
10. Thomas Jech, Set theory, Springer Monographs in Mathematics, Springer-Verlag, Berlin, 2003, The third millennium edition, revised and expanded. MR MR1940513 (2004g:03071)

11. Thomas Jech and Karel Prikry, On ideals of sets and the power set operation, Bull. Amer. Math. Soc. 82 (1976), no. 4, 593-595. MR MR0505504 (58 \#21618)

12. Thomas J. Jech, More game-theoretic properties of Boolean algebras, Ann. Pure Appl. Logic 26 (1984), no. 1, 11-29. MR MR739910 (85j:03110)

13. Some properties of $\kappa$-complete ideals defined in terms of infinite games, Ann. Pure Appl. Logic 26 (1984), no. 1, 31-45. MR MR739911 (85h:03057)

14. Thomas J. Jech and William J. Mitchell, Some examples of precipitous ideals, Ann. Pure Appl. Logic 24 (1983), no. 2, 131-151. MR MR713297 (85k:03033)

15. Jakob Kellner, Matti Pauna, and Saharon Shelah, Winning the pressing down game but not Banach-Mazur, J. Symbolic Logic 72 (2007), no. 4, 1323-1335. MR MR2371208

16. Richard Laver, Making the supercompactness of $\kappa$ indestructible under $\kappa$-directed closed forcing, Israel J. Math. 29 (1978), no. 4, 385-388. MR MR0472529 (57 \#12226)

17. Alan Mekler, Saharon Shelah, and Jouko Väänänen, The Ehrenfeucht-Fraïssé-game of length $\omega_{1}$, Trans. Amer. Math. Soc. 339 (1993), no. 2, 567-580. MR MR1191613 (94a:03058)

18. Ernest Schimmerling and Boban Velickovic, Collapsing functions, MLQ Math. Log. Q. 50 (2004), no. 1, 3-8. MR MR2029599 (2005a:03100)

19. Boban Veličković, Playful Boolean algebras, Trans. Amer. Math. Soc. 296 (1986), no. 2, 727740. MR MR846604 (88a:06017)

20. Jindřich Zapletal, More on the cut and choose game, Ann. Pure Appl. Logic 76 (1995), no. 3, 291-301. MR MR1366514 (97b:03067)

21. Martin Zeman, Inner models and large cardinals, de Gruyter Series in Logic and its Applications, vol. 5, Walter de Gruyter \& Co., Berlin, 2002. MR MR1876087 (2003a:03004)

Kurt Gödel Research Center for Mathematical Logic, Universität Wien, Währinger Strasse 25, 1090 Wien, Austria

E-mail address: kellner@fsmat.at

$U R L:$ http://www.logic.univie.ac.at/ kellner

Einstein Institute of Mathematics, Edmond J. Safra Campus, Givat Ram, The Hebrew University of Jerusalem, Jerusalem, 91904, Israel, and Department of Mathematics, Rutgers University, New Brunswick, NJ 08854, USA

E-mail address: shelah@math.huji.ac.il

URL: http://shelah.logic.at/ 